\title{
Fingerprint Classification by Directional Image Partitioning
}

\author{
Raffaele Cappelli, Alessandra Lumini, \\ Dario Maio, Member, IEEE, and Davide Maltoni
}

\begin{abstract}
In this work, we introduce a new approach to automatic fingerprint classification. The directional image is partitioned into "homogeneous" connected regions according to the fingerprint topology, thus giving a synthetic representation which can be exploited as a basis for the classification. A set of dynamic masks, together with an optimization criterion, are used to guide the partitioning. The adaptation of the masks produces a numerical vector representing each fingerprint as a multidimensional point, which can be conceived as a continuous classification. Different search strategies are discussed to efficiently retrieve fingerprints both with continuous and exclusive classification. Experimental results have been given for the most commonly used fingerprint databases and the new method has been compared with other approaches known in the literature: As to fingerprint retrieval based on continuous classification, our method gives the best performance and exhibits a very high robustness.
\end{abstract}

Index Terms-Fingerprint classification, directional image, partitioning algorithms, continuous classification, biometric systems.

\section{INTRODUCTION}

$\mathrm{F}$ INGERPRINT recognition is the basic task of the Integrated Automated Fingerprint Identification Service (IAFIS) of the most famous police agencies. Ten-print based identification and latent fingerprint recognition are the two main concerns of an IAFIS. In the former, the system should identify a person by the whole sequence of his/her 10 fingerprints; in the latter, it has to identify a person through a latent fingerprint found on a crime scene. The huge amount of data of the large fingerprint databases seriously compromises the efficiency of the identification task, although the fastest minutiae matching algorithms take only a few tens of milliseconds per matching [15]. Adopting a classification approach, based on common typology of fingerprints, is a valid strategy in order to reduce the amount of matching during fingerprint retrieval and, consequently, to improve the identification process efficiency.

Most IAFISs use exclusive classification, i.e., fingerprints are partitioned into some predefined classes according to their macro-features. The first scientific studies on fingerprint classification were made by Galton [7], who divided the fingerprints into three major classes. Later, Henry [8] refined Galton's classification by increasing the number of the classes. All the classification schemes currently used by police agencies are variants of the so-called Henry's classification scheme. The scheme adopted by the FBI defines three major classes, each of which can be divided into two or more subclasses, making the total number eight: Plain Arch,

- R. Cappelli is with Corso di Laurea in Scienze dell'Informazione, Università di Bologna, via Sacchi 3, 47023 Cesena, Italy. E-mail: cappelli@csr.unibo.it.

- A. Lumini, D. Maio, and D. Maltoni are with DEIS, CSITE-CNR, Università di Bologna, viale Risorgimento 2, 40136 Bologna, Italy.

E-mail: \{alumini,dmaio,dmaltoni\}@deis.unibo.it.

Manuscript received 31 July 1998; revised 9 Feb. 1999. Recommended for acceptance by K. Bowyer.

For information on obtaining reprints of this article, please send e-mail to: tpami@computer.org, and reference IEEECS Log Number 107372.
Tented Arch, Radial Loop, Ulnar Loop, Plain Whorl, Central Pocket, Double Loop and Accidental Whorl (Fig. 1).

The performance of the exclusive classification-based IAFISs strongly depends on the number of classes and on the distribution of fingerprints; unfortunately, the number of classes is often small, the fingerprints are nonuniformly distributed (in the most famous classification schemes, approximately 90 percent of fingerprints belong to only three classes), and there are many "ambiguous" fingerprints, whose exclusive membership cannot be reliably stated even by human experts. Nevertheless, exclusive classification allows for the efficiency of the 10-print based identification to be improved, since the knowledge of the classes of the ten fingerprints can be used as a code for reducing the number of comparisons at minutiae level. On the other hand, an exclusive classification approach does not confer sufficient selectivity to latent fingerprint searching.

A continuous classification approach characterizes each fingerprint with a numerical vector, whose components denote the similarity degree with respect to a predefined set of class prototypes. A continuous approach enables both ambiguous fingerprints to be dealt with and the efforts during fingerprint retrieval to be balanced. The advantages of a continuous versus an exclusive classification approach are discussed in [16], where some continuous retrieval strategies were compared with the corresponding exclusive ones.

In this work, we present a new fingerprint classification method which uses dynamic masks for directional image partitioning. The new approach is translation and rotation invariant and it does not require the singularities [25] to be detected. A directional image is a discrete matrix whose elements represent the local average directions of the fingerprint ridge lines. A directional image effectively summarizes the information contained in a fingerprint pattern and it can be reliably computed also on noisy fingerprints. Furthermore, the local directions within a damaged area can be 

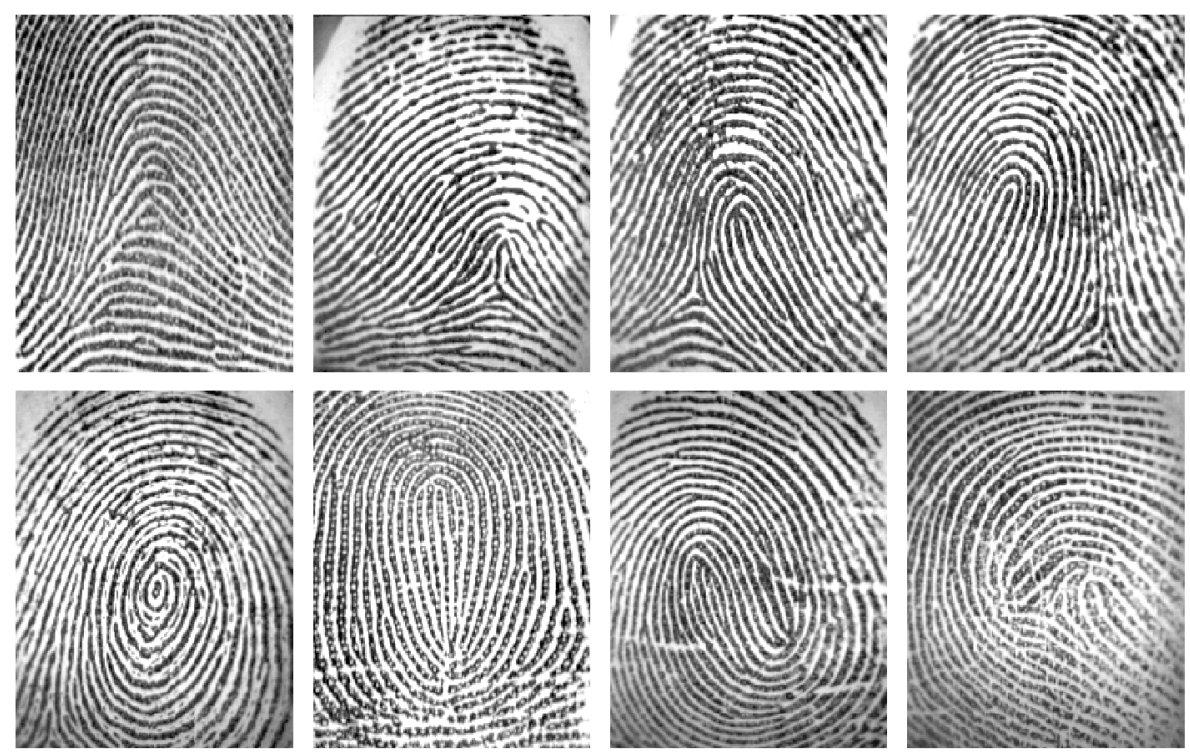

Fig. 1. Each fingerprint in the figure belongs to a different subclass of the FBl's classification scheme; from left to right and from top to bottom: Plain Arch, Tented Arch, Radial Loop, Ulnar Loop, Plain Whorl, Central Pocket, Double Loop, and Accidental Whorl.

partially restored by means of a regularization process. For the above reasons, most of the existing classification methods make use of fingerprint directional images. The basic idea of the method presented here consists of generating a compact version of the directional image by grouping similar elements into "homogeneous" regions. In fact, evident redundancy usually affects fingerprint patterns due to the smoothness of the ridge lines; hence, partitioning into homogeneous regions gives a useful synthetic representation which can be exploited as a basis for the classification task. Actually, the method is an evolution of the approach based on relational graphs [18], aimed at overcoming the problems found by the authors during the experimentation.

In Section 2, the main classification approaches known in the literature are reported; particular emphasis is given to the method PCASYS [4] and to a corresponding continuous implementation [16]; furthermore, the method [18] which constitutes an initial approach to fingerprint classification by directional image partitioning is briefly summarized. Section 3 introduces the new method and, in particular, it formally defines the concept of dynamic mask and the cost measure used to obtain an optimal partitioning. In Section 4, the retrieval strategies introduced in [16] are briefly reviewed, since they are adopted in this work to evaluate the classification performance. Section 5 describes some experimental results for NIST Database 4 [27] and NIST Database 14 [28]. As to continuous classification, our method is compared with that presented in [16]; although the new method was originally conceived for continuous classification and its evolution accompanied such a choice, it can also be applied to exclusive classification: Some comparisons with [4] and [13] are included in Section 5. The experimental results show the superiority of continuous classification for the retrieval task, where the approach presented here exhibits better performance than [16]. As to exclusive classification, the performance of our method is not much worse than those reported in [4] and [13], which were specifically developed for this task and include ad hoc heuristics to improve the classification accuracy. Furthermore, the robustness of the method has been evaluated over a set of manually deformed or deteriorated fingerprint images; the results reported at the end of Section 5 prove that it is usually very tolerant with respect to poor quality fingerprints. Finally, Section 6 draws some concluding remarks and includes proposals of future work.

\section{Literature ReView}

Many approaches to automatic fingerprint classification have been presented in the literature and the research on this topic is still very active. The existing methods can be coarsely assigned to one of the following categories: syntactic methods, approaches based on singularities, neural approaches, and others.

- Syntactic methods [20], [22]. Patterns are described by means of terminal symbols and production rules. Terminal symbols are associated to small groups of directional elements within the fingerprint directional image. A grammar is defined for each class and a parsing process is responsible for classifying each new pattern.

- Approaches based on singularities [13], ,14]. Heuristic criteria based on the number and the position of the singularities [15] are used to classify fingerprints. Other local features, such as ridge line shape and local orientation, are exploited to improve the performance. Since these methods heavily rely on singularities, some problems arise in the presence of noisy or partial fingerprints, where singularity detection can be misleading. In [13] an iterative regularization is carried out, until a valid number of singular points is detected, to reduce noise and consequently to improve the classification accuracy. 


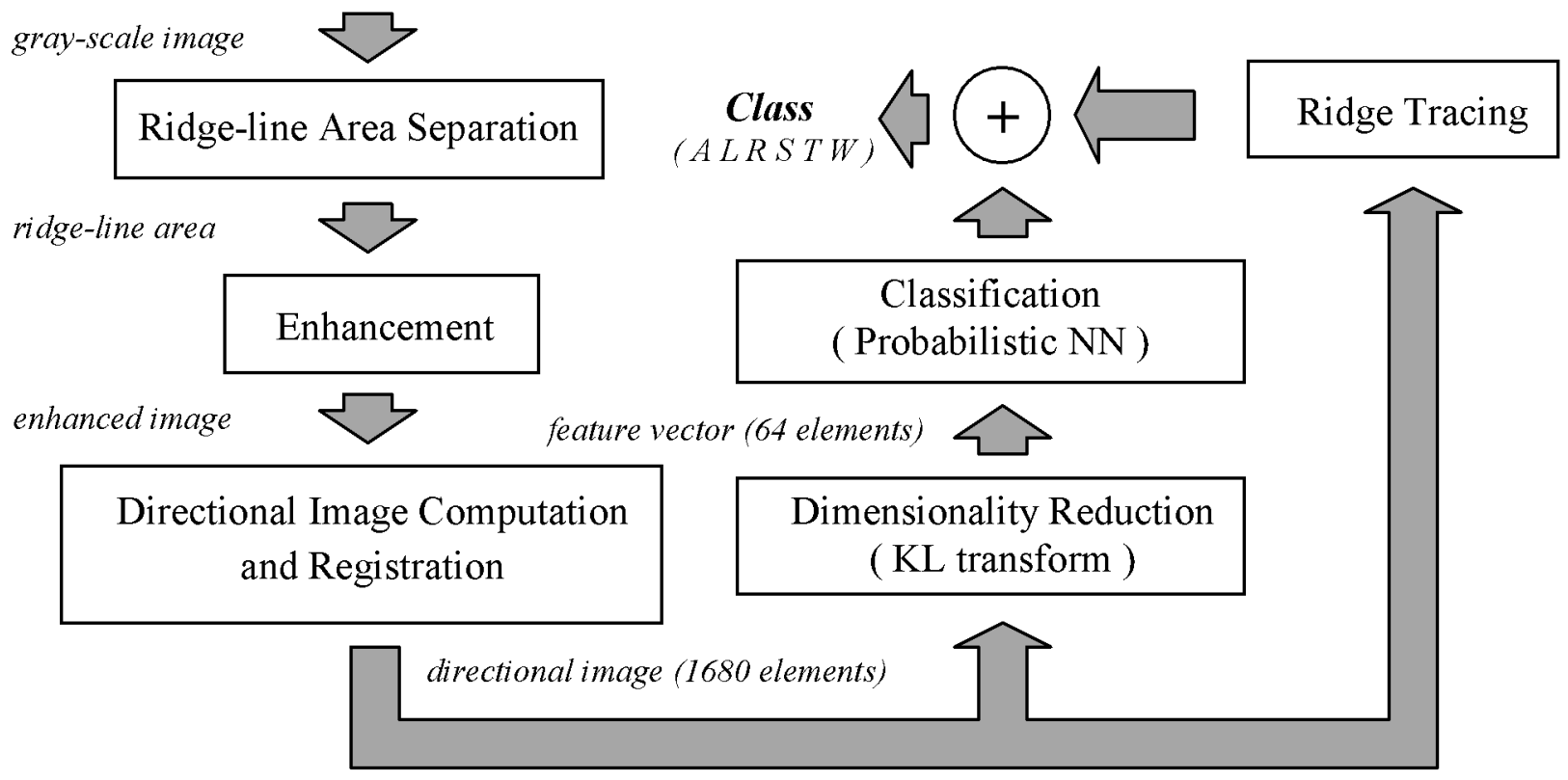

Fig. 2. A functional scheme of the PCASYS for exclusive classification.

- Neural approaches [1], [9], [12], [21]. Neural network approaches are mostly based on multilayer perceptrons or Kohonen self-organizing networks. In particular, in [12], Kamijo presented an interesting pyramidal architecture constituted by several multilayer perceptrons, each of which was trained to recognize fingerprints belonging to different classes.

- Others [5], [23]. Chong et al. based the classification on the ridge-line geometrical shape [5]. B-spline curves were used to model fingerprint ridge lines; adjacent curves were merged to limit noise artifacts. Classification was performed by tracing the resulting curves in order to detect turns (i.e., complete direction exchanges). Senior proposed in [23] a hidden Markov model classifier whose input features are the measurements (ridge angle, separation, curvature, etc.) taken at the intersection points between some horizontal-vertical fiducial lines and the fingerprint ridge lines.

\subsection{The PCASYS Approach by NIST}

The PCASYS approach (Pattern-level Classification Automation SYStem) proposed by Candela et al. at NIST [3], [4] assigns fingerprints to six nonoverlapping classes and is, in our opinion, the most promising approach for exclusive classification.

Before computing the directional images, the ridge-line area is separated from the background and an enhancement is performed in the frequency domain. The computation of the directions is carried out by the method reported in [26]. The directional image is then registered with respect to the core position which corresponds to the fingerprint center. The dimensionality of the directional image, considered as a vector of 1,680 elements, is reduced to 64 elements by using the principal component analysis (KL transform) [10], [11]. At this stage, a PNN (Probabilistic Neural Network) [24] is used for assigning each 64-element vector to one class of the classification scheme. In order to improve the classification reliability, especially for whorl fingerprints, the authors also implemented an auxiliary module (called pseudoridge tracer), which works by analyzing the ridge-line concavity under the core position. Fig. 2 shows a functional scheme of the NIST classification approach.

In [16], a continuous classification method is derived from PCASYS. In particular, the vectors obtained after the dimensionality reduction step are directly used for indexing a fingerprint database through a spatial data structure. In the experiments the authors found that the best results could be obtained by using a five-dimensional space (instead of 64) and by linearly rescaling the vector components in the range $[0,1]$.

\subsection{A Structural Approach Based on Relational Graphs}

In [18], a structural approach for fingerprint classification was presented, whose functional schema is shown in Fig. 3.

The basic idea is to perform a directional image partitioning into several homogeneous regular-shaped regions, which are used to build a relational graph summarizing the fingerprint macro-features. The whole approach can be divided into four main steps: computation of the directional image, segmentation of the directional image, construction of the relational graph, and inexact graph matching. The directional image is computed, over a discrete grid $32 \times 32$, by means of a robust technique proposed by Donahue and Rokhlin [6]. A dynamic clustering algorithm [19] is adopted to segment the directional image according to well-suited optimality criteria. In particular, with the aim of creating regions as homogeneous as possible, the algorithm works by minimizing the variance of the element directions within the regions and, simultaneously, by maintaining the regularity of the region shape. Starting from the segmentation of the directional image, a relational graph is built by creating a node for each region and an arc for each pair of adjacent 

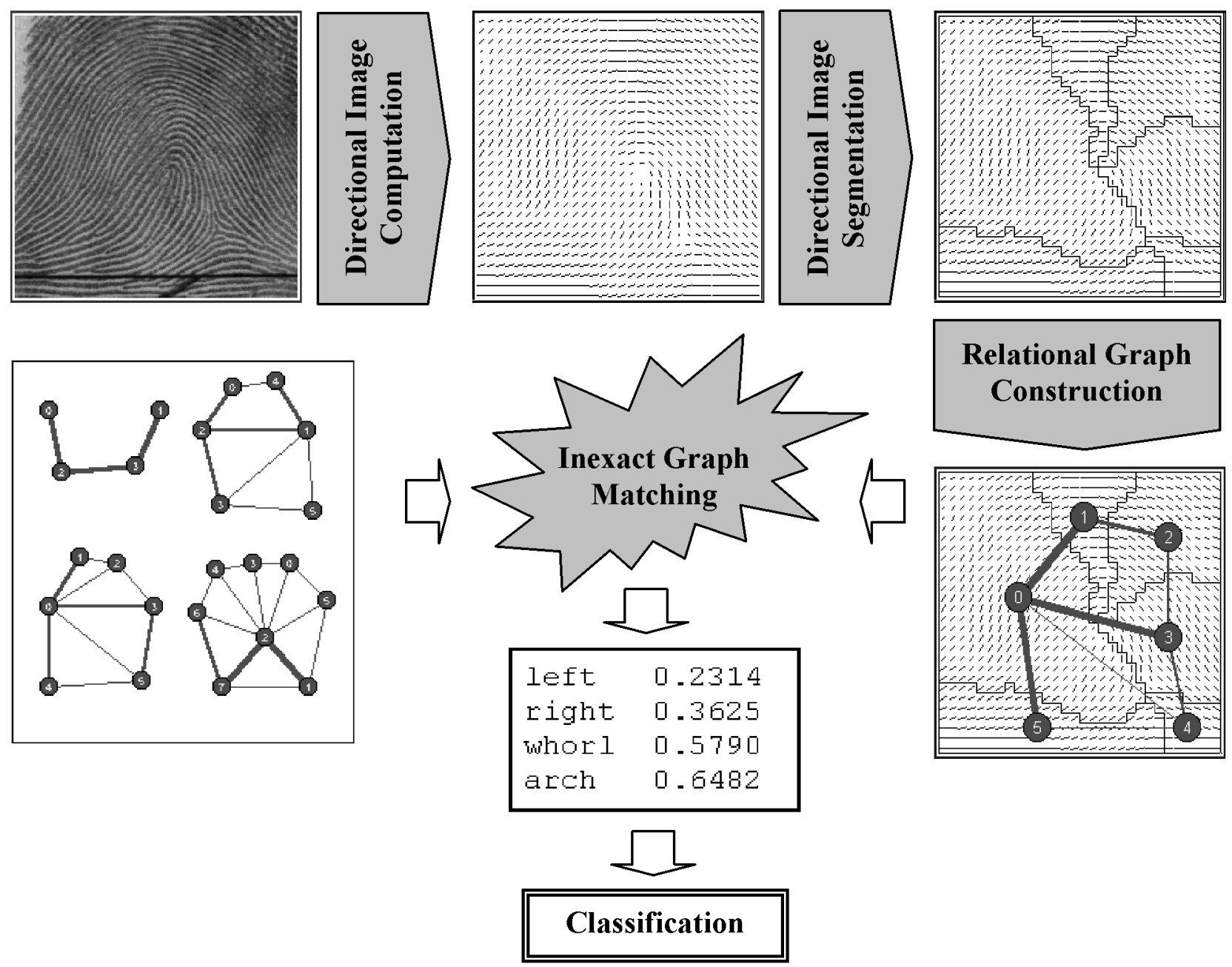

Fig. 3. The main steps of the approach in [18]. The intermediate results produced during the classification of a Left Loop fingerprint are shown.

regions. By appropriately labeling the nodes and arcs of the graph, the authors obtain a structure which summarizes the topological features of the fingerprint and which is invariant with respect to displacement and rotation. An inexact graph matching technique derived from [2] is used to compute a "distance" vector between the graph and each classprototype graph. This distance is considered to be a continuous classification of the fingerprint, since it describes how close the fingerprint is to each class; if the application needs to assign a fingerprint to a single class, the vector obtained can be given as input to a general purpose classifier. It is worth noting that this technique does not require any position alignment or normalization and, in principle, can be directly used for the classification of partial fingerprints (i.e., matching a graph with a subgraph).

\section{DYNAMIC MASKS}

\subsection{From Relational Graphs to Dynamic Masks}

A preliminary analysis of the experimental results produced by the relational graph approach described in Section 2.2 pointed out that some problems arise from the difficulty in obtaining analogous segmentation from similar directional images. In fact, although the greedy clustering algorithm adopted usually produces a good segmentation (that is, characterized by regular-shaped and homogeneous regions), it is too influenced by local ridge-line orientation changes, by the starting point of the clustering routines, and by some specific parameters; as a result, similar inputs can give rise to different outputs (Fig. 4). In such cases, several problems are encountered by the inexact graph matching algorithm in finding the hidden similarities. An attempt was made to overcome these drawbacks by implementing a global clustering strategy, with the aim of improving the segmentation robustness. A genetic algorithm derived from the approach [17], where reproduction and mutation operators were redesigned to deal with the clustering problem, was used instead of the greedy technique. Better results were obtained both in qualitative and numerical terms (obviously with a higher computational cost) and a greater robustness was noted (see Fig. 5 for an example); anyway, some problems still persisted: In fact, the outputs produced by the genetic optimization in the presence of different initial populations were sometimes very different from each other, even if their fitness was closed. This clearly means that the directional image partitioning task is an ill-posed problem. 

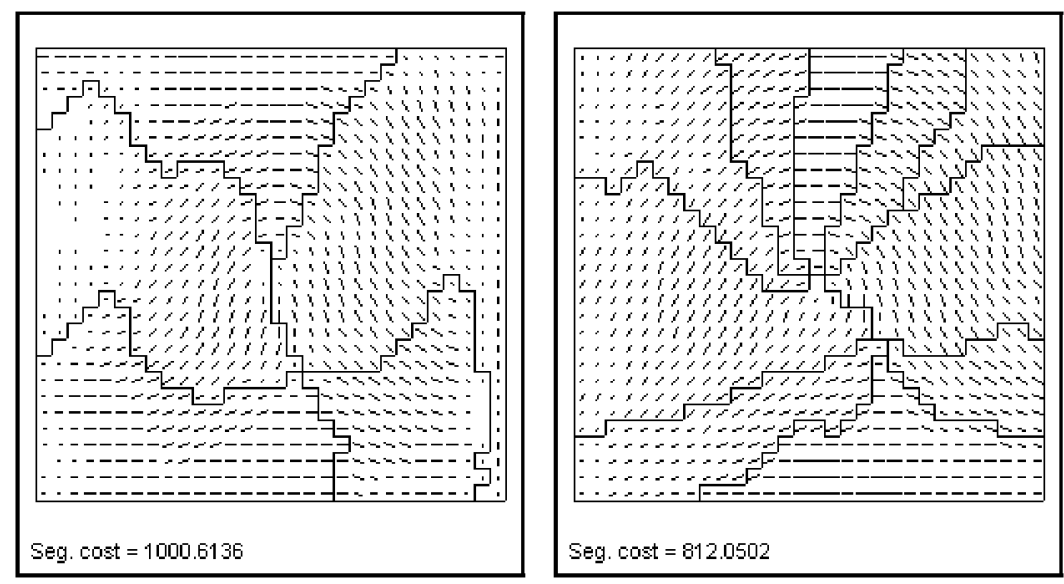

Fig. 4. The segmentation of two Left Loop fingerprints.
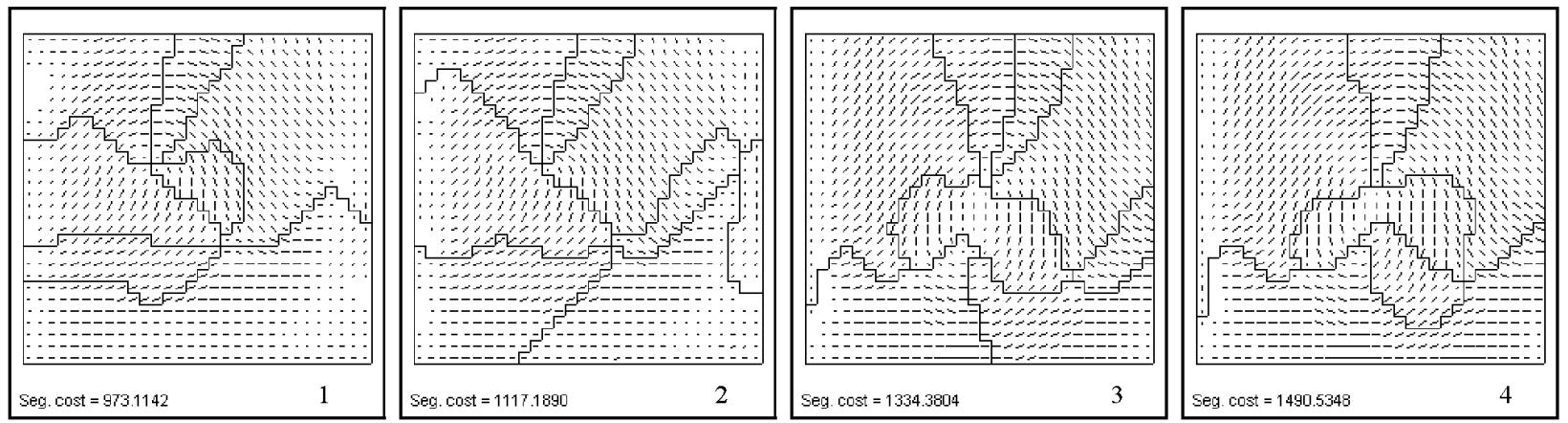

Fig. 5. Segmentation results given by the genetic approach ( 1 and 3 ) and by the greedy strategy (2 and 4$)$ starting from the same input images $(1 \equiv 2,3 \equiv 4)$.

\subsection{An Overview of the New Approach}

The basic idea of the new approach is to perform a "guided" segmentation of the directional image with the aim of drastically reducing the degrees of freedom during the partitioning process and, consequently, conferring stability to the solutions. To this purpose, a set of dynamic masks, directly derived from the most common fingerprint classes, are used to guide the partitioning. Profound modifications result in the approach: In particular, the inexact graph matching step is simplified and embedded in the segmentation step. Fig. 6 reports the schema of the new method applied to a Left Loop fingerprint: The directional image is initially calculated and enhanced; a set of dynamic masks is used for the segmentation step where each mask is independently adapted to best fit the directional image according to a cost function; the resulting costs constitute a basis for the final classification.

\subsection{Directional Image Computation and Enhancement}

The fingerprint segmentation, preprocessing, and directional image computation are performed as in [4]: The finger area is separated from the background and its quality is improved by a filtering in the frequency domain; then, the Stock and Swonger method [26] is applied to calculate directional elements over a discrete grid $28 \times 30$. Each element is denoted by a vector $\mathbf{v}=[\xi \cos 2 \theta, \xi \sin 2 \theta]$, where $\theta \in\left[0^{\circ}, 180^{\circ}\right)$ represents the orientation and $|\mathbf{v}|=\xi$ gives a confidence value. This kind of encoding allows the problems induced by the orientation discontinuity $\left(180^{\circ} \leftrightarrow 0^{\circ}\right)$ to be avoided [4].

In order to smooth out the local irregularities produced by noise and to increase the importance of the discriminant elements, the following three steps are carried out on the directional image:

- Regularization of the directional elements by local averaging on $3 \times 3$ windows $W$.

$$
\mathbf{v}_{\mathrm{r}}=\frac{1}{9} \sum_{\mathbf{v} \in \mathrm{W}_{3 \times 3}} \mathbf{v}
$$

- Attenuation of the border elements by applying a Gaussian-like function which progressively reduces the element magnitude moving from the center towards the borders. The attenuation function att is defined as:

$$
\operatorname{att}(\mathbf{v})=\frac{1}{\sqrt{2 \pi \cdot \sigma}} \mathrm{e}^{-\frac{\operatorname{distc}(\mathbf{v})^{2}}{2 \sigma^{2}}}
$$

where $\operatorname{distc}(\mathbf{v})$ returns the distance in blocks of $\mathbf{v}$ origin from the directional image center and $\sigma$ determines the scale of the Gaussian function.

- Strengthening of the directional elements located in the irregular regions mainly determined by the fingerprint singularities. To this purpose, we use a strengthening function (str) which increases the 

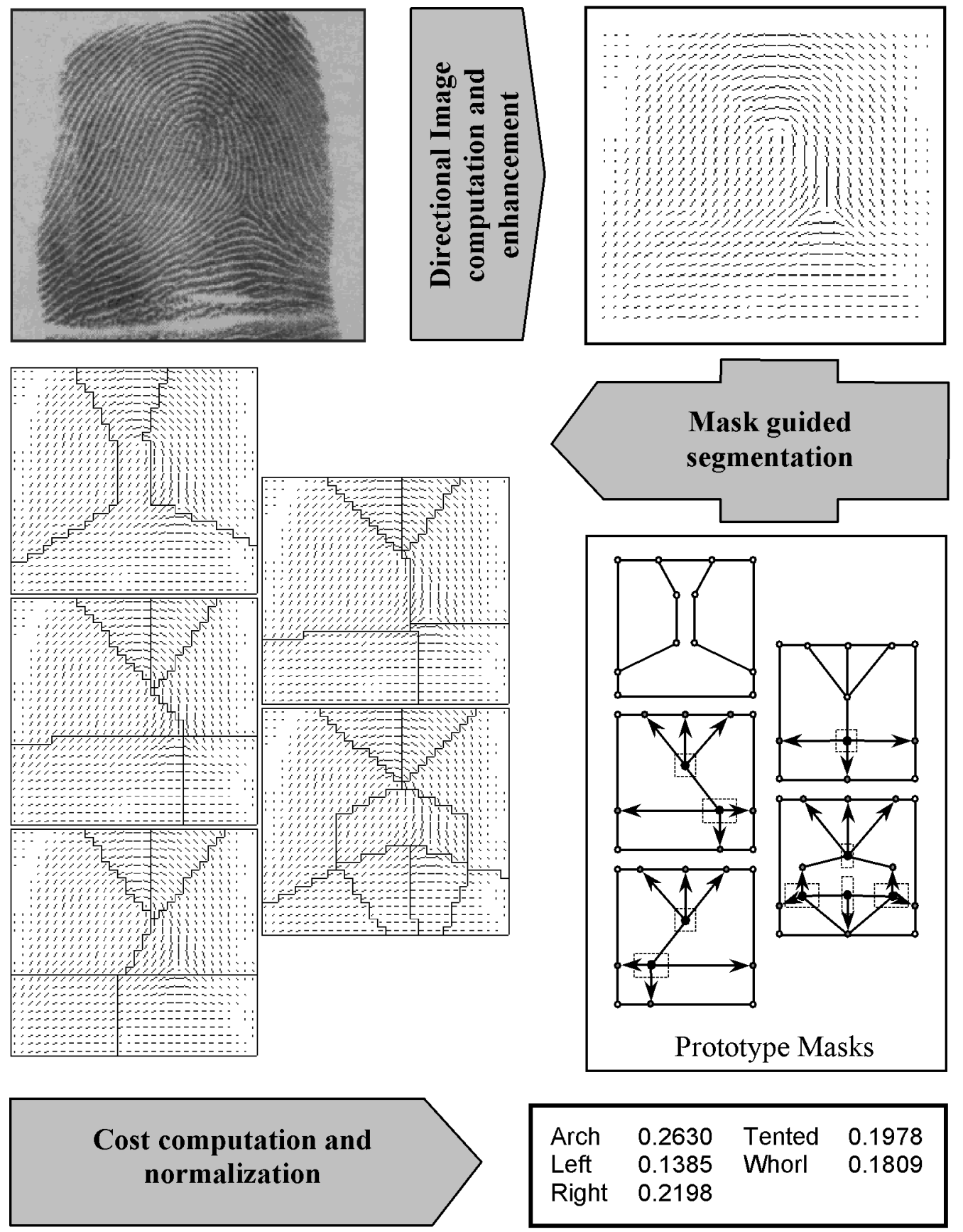

\begin{tabular}{|llll|}
\hline Arch & 0.2630 & Tented & 0.1978 \\
Left & 0.1385 & Whorl & 0.1809 \\
Right & 0.2198 & & \\
\hline
\end{tabular}

Fig. 6. Classification of a Left Loop fingerprint by means of the dynamic masks approach.

significance of each element according to the irregularity degree of its $3 \times 3$ neighborhood, without requiring the singularities to be explicitly detected. For each element, a local $3 \times 3$ window is considered and a strengthening function str is applied. str is defined as one minus the magnitude of the sum of the directional elements divided by the sum of element magnitudes; it returns 0 if all the vectors are parallel to each other and its value approaches 1 when discordance increases.

$$
\operatorname{str}(\mathbf{v})=1-\frac{\mid \sum_{\mathbf{v} \in \mathrm{W}_{3 \times 3} \mathbf{v} \mid}}{\sum_{\mathbf{v} \in \mathrm{W}_{3 \times 3}|\mathbf{v}|}}
$$

The resulting directional image is made up of vectors $\mathbf{v}_{\mathrm{e}}$ such that:

$$
\mathbf{v}_{\mathrm{e}}=\mathbf{v}_{\mathrm{r}} \cdot\left(1+\gamma \cdot \operatorname{att}\left(\mathbf{v}_{\mathrm{r}}\right) \cdot \frac{1}{9} \sum_{\mathbf{v}_{\mathrm{r}} \in \mathrm{W}_{3 \times 3}} \operatorname{str}\left(\mathbf{v}_{\mathrm{r}}\right)\right),
$$

where $\gamma$ is a weighting factor. 

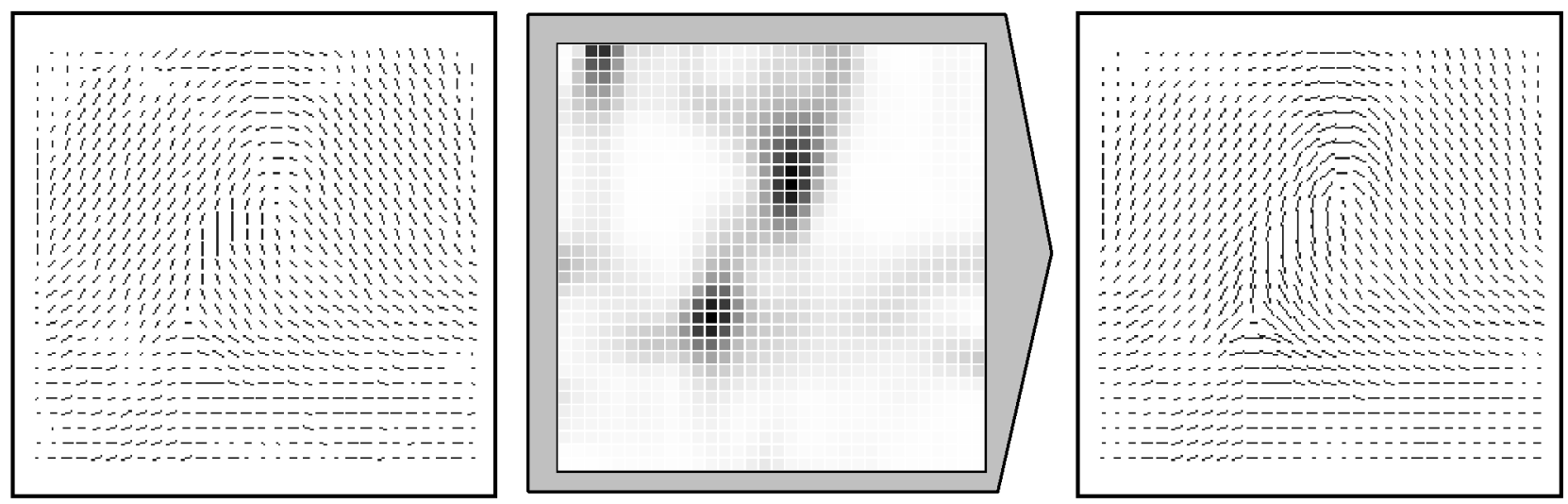

Fig. 7. Enhancement of a directional image: the map in the arrow-box shows the most irregular regions as revealed by the str function. The parameters are: $\sigma=9.6$ and $\lambda=112$.
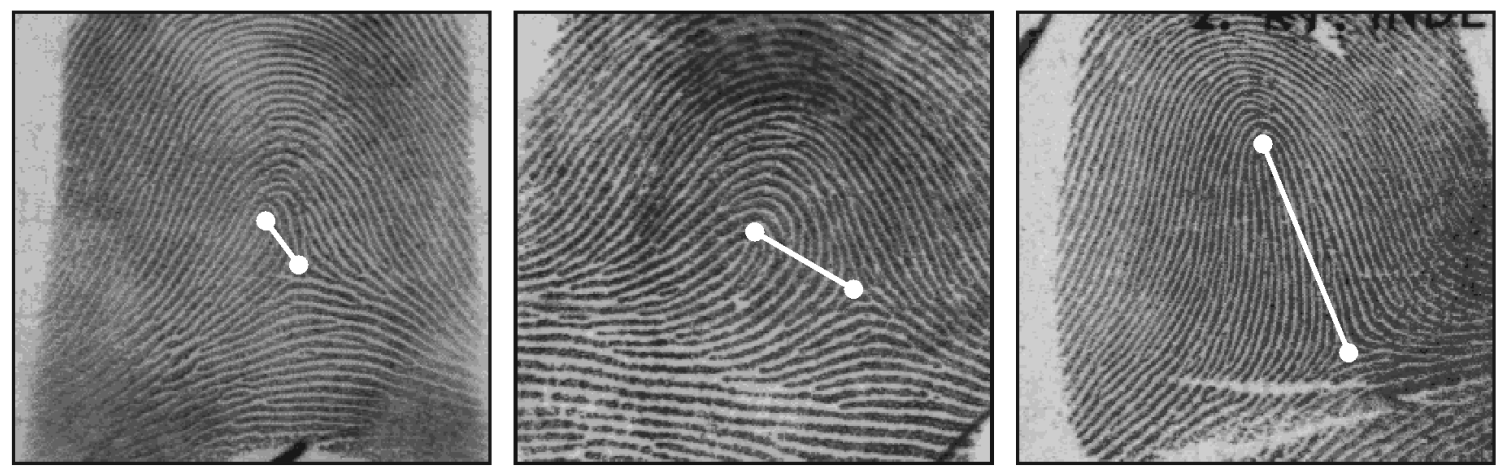

Fig. 8. The singularity positions in three different Left Loop fingerprints.

Fig. 7 shows a sample directional image before and after the enhancement process.

\subsection{Dynamic Mask Definition}

Dynamic masks have been introduced in order to decrease the degrees of freedom during the partitioning process; the application of a particular mask to a directional image can be viewed as a "guided" segmentation, where the region number and their coarse shape are fixed a priori. Each mask is characterized by a set of vertices defining the borders of the regions which determine the segmentation. Some vertices can be locally moved to best fit the fingerprint image singularities, which can occupy different positions within fingerprints of the same class (Fig. 8).

Formally, a dynamic mask is defined as a 6-tuple $\mathrm{M}=$ $\left(\mathrm{V}, \mathrm{P}, \mathcal{R}_{\mathrm{D}}, \mathcal{A}, f_{\text {win }} f_{\text {mov }}\right)$, where:

- $\mathrm{V}=\mathrm{V}_{\mathrm{F}} \cup \mathrm{V}_{\mathrm{M}} \cup \mathrm{V}_{\mathrm{D}}$ is a set of vertices $\mathbf{p}$, each of which is characterized by an initial position $\left(\mathrm{p}_{\mathrm{x}}, \mathrm{p}_{\mathrm{y}}\right) ; \mathrm{V}_{\mathrm{F}}$ denotes the fixed vertices; $\mathrm{V}_{\mathrm{M}}$ denotes the mobile vertices whose position can be independently adjusted during the mask adaptation; finally, $\mathrm{V}_{\mathrm{D}}$ denotes the dependent vertices whose position is anchored to that of one mobile vertex. The three vertex subsets are disjointed: $\mathrm{V}_{\mathrm{F}} \cap \mathrm{V}_{\mathrm{M}}=\varnothing, \mathrm{V}_{\mathrm{F}} \cap \mathrm{V}_{\mathrm{D}}=\varnothing, \mathrm{V}_{\mathrm{D}} \cap \mathrm{V}_{\mathrm{M}}=\varnothing$.

- $\mathrm{P}=\left\{\mathrm{P}_{1}, \mathrm{P}_{2}, \ldots, \mathrm{P}_{\mathrm{n}}\right\}$ is a set of polygonal regions whose vertices are in $V$; each region $P_{i}=\left\{p_{a}, p_{b}, p_{c}, \ldots\right\}$ is bounded by the polygon defined by the vertices $\mathbf{p}_{\mathrm{a}}, \mathbf{p}_{\mathrm{b}}, \mathbf{p}_{\mathrm{c}}, \ldots$ taken in the given order; hence, $\mathrm{P}$ is a subset of the power set of $\mathrm{V}: \mathrm{P} \subset \wp(\mathrm{V})$.
- $\mathcal{R}_{\mathrm{D}} \subseteq \mathrm{V}_{\mathrm{D}} \times \mathrm{V}_{\mathrm{M}}$ is a relation encoding the dependency of the dependent vertices from the mobile ones. Each dependent vertex is anchored to exactly one mobile vertex; during the mask adaptation, the movement of a mobile vertex determines a corresponding movement in all the vertices anchored to it.

- $\mathcal{A} \subseteq \mathrm{P} \times \mathrm{P} \times \Delta_{\theta}$ encodes a relation between some region pairs, which are associated to angles representing the "ideal" values of their mean orientation difference. $\Delta_{\theta}$ denotes the domain of the orientation differences. For each pair $P_{i_{1}} P_{j}$, whose orientation difference $\theta_{\mathrm{ij}} \in \Delta \theta$ is significant, the triplet $\left(\mathrm{P}_{\mathrm{i}}, \mathrm{P}_{\mathrm{j}}, \theta_{\mathrm{ij}}\right) \in \mathcal{A}$.

- $f_{\text {win }}: \mathrm{V}_{\mathrm{M}} \rightarrow \Delta_{\mathrm{xmax}} \times \Delta_{\mathrm{ymax}}$ is a function which associates to each mobile vertex a mobility window which limits the vertex movements during the mask adaptation. $\Delta_{\mathrm{xmax}}$ and $\Delta_{\mathrm{ymax}}$ represent the domain of maximum displacements along the $\mathrm{x}$ and $\mathrm{y}$ axes, respectively.

- $f_{\text {mov }}: \mathcal{R}_{\mathrm{D}} \times \Delta_{\mathrm{x}} \times \Delta_{\mathrm{y}} \rightarrow \Delta_{\mathrm{x}} \times \Delta_{\mathrm{y}}$ is a function which indicates, for each pair in $\mathcal{R}_{\mathrm{D}}$, the dependent vertex movement on the basis of the corresponding mobile vertex movement. $\Delta_{\mathrm{x}}$ and $\Delta_{\mathrm{y}}$ represent the domain of displacements along $\mathrm{x}$ and $\mathrm{y}$ axes, respectively.

Fig. 9 shows an example.

1. An orientation difference is significant if it is useful to discriminate fingerprints belonging to different classes. 


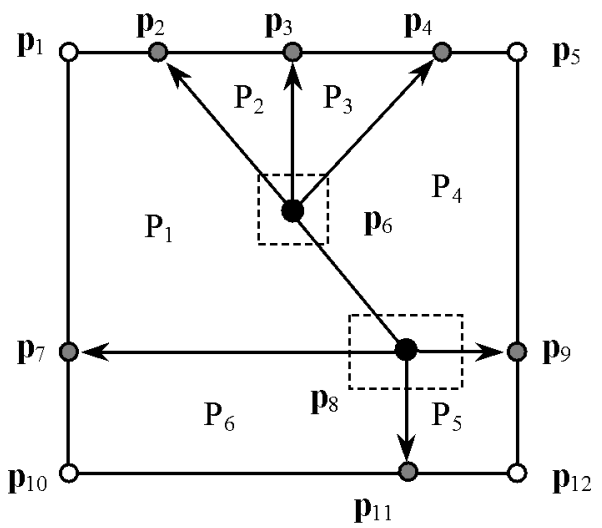

$$
\begin{aligned}
& \mathrm{V}_{\mathrm{F}}=\left\{\mathbf{p}_{1}, \mathbf{p}_{5}, \mathbf{p}_{10}, \mathbf{p}_{12}\right\}, \mathrm{V}_{\mathrm{M}}=\left\{\mathbf{p}_{6}, \mathbf{p}_{8}\right\}, \mathrm{V}_{\mathrm{D}}=\left\{\mathbf{p}_{2}, \mathbf{p}_{3}, \mathbf{p}_{4}, \mathbf{p}_{7}, \mathbf{p}_{9}, \mathbf{p}_{11}\right\} \\
& \mathbf{P}=\left\{\mathbf{P}_{1}=\left\{\mathbf{p}_{1}, \mathbf{p}_{2}, \mathbf{p}_{6}, \mathbf{p}_{8}, \mathbf{p}_{7}\right\}, \mathbf{P}_{2}=\left\{\mathbf{p}_{2}, \mathbf{p}_{3}, \mathbf{p}_{6}\right\}, \mathrm{P}_{3}=\left\{\mathbf{p}_{3}, \mathbf{p}_{4}, \mathbf{p}_{6}\right\},\right. \\
& \left.\mathbf{P}_{4}=\left\{\mathbf{p}_{4}, \mathbf{p}_{5}, \mathbf{p}_{9}, \mathbf{p}_{6}\right\}, \mathbf{P}_{5}=\left\{\mathbf{p}_{8}, \mathbf{p}_{9}, \mathbf{p}_{12}, \mathbf{p}_{11}\right\}, \mathbf{P}_{6}=\left\{\mathbf{p}_{7}, \mathbf{p}_{8}, \mathbf{p}_{11}, \mathbf{p}_{10}\right\}\right\} \\
& \mathcal{R}_{\mathrm{D}}=\left\{\left(\mathbf{p}_{2}, \mathbf{p}_{6}\right),\left(\mathbf{p}_{3}, \mathbf{p}_{6}\right),\left(\mathbf{p}_{4}, \mathbf{p}_{6}\right),\left(\mathbf{p}_{7}, \mathbf{p}_{8}\right),\left(\mathbf{p}_{9}, \mathbf{p}_{8}\right),\left(\mathbf{p}_{11}, \mathbf{p}_{8}\right)\right\} \\
& \mathcal{A}=\left\{\left(\mathrm{P}_{1}, \mathrm{P}_{6}, 36^{\circ}\right)\right\} \\
& f_{\text {win }}\left(\mathbf{p}_{6}\right)=(4,4), f_{\text {wim }}\left(\mathbf{p}_{8}\right)=(6,4) \\
& f_{\text {mov }}\left(\left(\mathbf{p}_{2}, \mathbf{p}_{6}\right), \mathrm{d}_{\mathrm{x}}, \mathrm{d}_{\mathrm{y}}\right)=\left(\mathrm{d}_{\mathrm{x}}, 0\right), f_{\mathrm{mov}}\left(\left(\mathbf{p}_{3}, \mathbf{p}_{6}\right), \mathrm{d}_{\mathrm{x}}, \mathrm{d}_{\mathrm{y}}\right)=\left(\mathrm{d}_{\mathrm{x}}, 0\right), \\
& f_{\text {mov }}\left(\left(\mathbf{p}_{4}, \mathbf{p}_{6}\right), \mathrm{d}_{\mathrm{x}}, \mathrm{d}_{\mathrm{y}}\right)=\left(\mathrm{d}_{\mathrm{x}}, 0\right), f_{\text {mov }}\left(\left(\mathbf{p}_{7}, \mathbf{p}_{8}\right), \mathrm{d}_{\mathrm{x}}, \mathrm{d}_{\mathrm{y}}\right)=\left(0, \mathrm{~d}_{\mathrm{y}}\right), \\
& f_{\text {mov }}\left(\left(\mathbf{p}_{9}, \mathbf{p}_{8}\right), \mathrm{d}_{\mathrm{x}}, \mathrm{d}_{\mathrm{y}}\right)=\left(0, \mathrm{~d}_{\mathrm{y}}\right), f_{\text {mov }}\left(\left(\mathbf{p}_{11}, \mathbf{p}_{8}\right), \mathrm{d}_{\mathrm{x}}, \mathrm{d}_{\mathrm{y}}\right)=\left(\mathrm{d}_{\mathrm{x}}, 0\right)
\end{aligned}
$$

Fig. 9. An example of dynamic mask definition. Fixed vertices are denoted by empty circles, the mobile ones by black circles, and the dependent ones by gray circles. The dashed boxes denote the mobility windows associated to the mobile vertices. An arrow from a mobile vertex $\mathbf{p}_{i}$ to a dependent vertex $\mathbf{p}_{\mathrm{j}}$ indicates the dependence of $\mathbf{p}_{\mathrm{j}}$ on $\mathbf{p}_{\mathrm{i}}$.

\subsection{Directional Image Partitioning With Dynamic Masks}

Let $\mathrm{M}_{\mathrm{T}, \mathrm{Q}}$ be the steady mask obtained by the dynamic mask $\mathrm{M}$ as a result of the following transformations:

- a global rotation-displacement $\mathrm{T}=\left(\delta_{\mathrm{x}}, \delta_{\mathrm{y}}, \varphi\right)$, where $\delta_{\mathrm{x}}$ and $\delta_{\mathrm{y}}$ denote the global mask displacement and $\varphi$ denotes the global mask rotation.

- a set of mobile vertex displacements $\mathrm{Q}=\left\{\left(\mathrm{d}_{\mathrm{x} 1}, \mathrm{~d}_{\mathrm{y} 1}\right)\right.$, $\left.\left(d_{x 2}, d_{y 2}\right), \ldots\right\} ;\left(d_{x i}, d_{y i}\right)$ denotes the displacement of the vertex $\mathbf{p}_{i}$ with respect to its initial position.

The application of a steady mask $\mathrm{M}_{\mathrm{T}, \mathrm{Q}}$ to a directional image $\mathrm{D}$ consists in superimposing $\mathrm{M}_{\mathrm{T}, \mathrm{Q}}$ on $\mathrm{D}$ and deriving $\mathrm{a}$ segmentation $R=\left\{R_{1}, R_{2}, \ldots, R_{n}\right\}$ where each region $R_{i}$ is made up of the directional elements internal to the polygon $\mathrm{P}_{\mathrm{i}}$.

A steady mask $M_{T, Q}$ well fits a directional image $D$ when the segmentation $R$ obtained by the application of $M_{T, Q}$ to $D$ is such that the orientations of the directional elements within each region $R_{i}$ are homogeneous and the orientation differences in $\mathcal{A}$ are close to the corresponding ones induced by $R$. Hence, the cost $C_{\mathrm{sm}}\left(\mathrm{M}_{\mathrm{T}, \mathrm{Q}}, \mathrm{D}\right)$ of the application of $\mathrm{M}_{\mathrm{T}, \mathrm{Q}}$ to $\mathrm{D}$ is given by the sum of two terms:

$$
\begin{aligned}
& C_{\mathrm{sm}}\left(\mathrm{M}_{\mathrm{T}, \mathrm{Q}}, \mathrm{D}\right)=\sum_{\mathrm{i}=1}^{\mathrm{n}}\left(\mathrm{C}_{0}+\operatorname{Var}\left(\mathrm{R}_{\mathrm{i}}\right)\right)+ \\
& \mu \cdot \frac{1}{\operatorname{card}(\mathcal{A})} \cdot \sum_{\left(\mathrm{P}_{\mathrm{i}}, \mathrm{P}_{\mathrm{j}}, \theta_{\mathrm{ij}}\right) \in \mathcal{A}} \Delta a b s\left(\operatorname{dir}\left(\mathrm{R}_{\mathrm{i}}, \mathrm{R}_{\mathrm{j}}\right), \theta_{\mathrm{ij}}\right),
\end{aligned}
$$

where:

- As to the first term, $\operatorname{Var}\left(\mathrm{R}_{\mathrm{i}}\right)$ is proportional to the variance of the directional elements in $R_{i}$ (see the Appendix) and $C_{0}$ is a parameter which introduces a penalty proportional to the number of regions in $\mathrm{M}$ in order to balance the possibility of obtaining lower costs by segmenting the directional image into several small regions. This cost coincides with the segmentation cost used in the relational graph approach in [18], except for the region-shape term which is now neglected due to the region shape fixed a priori.
- As to the second term, $\operatorname{\Delta dir}\left(\mathrm{R}_{\mathrm{i}}, \mathrm{R}_{\mathrm{j}}\right) \in\left[-90^{\circ}, 90^{\circ}\right)$ returns the difference between the average orientations $\theta_{\mathrm{i}}, \theta_{\mathrm{j}} \in$ $\left[-90^{\circ}, 90^{\circ}\right)$ of the regions $R_{i}$ and $R_{j}$ :

$$
\Delta \operatorname{dir}\left(R_{i}, R_{j}\right)= \begin{cases}\theta_{\mathrm{i}}-\theta_{\mathrm{j}} & \text { if }-90^{\circ} \leq \theta_{\mathrm{i}}-\theta_{\mathrm{j}}<90^{\circ} \\ \theta_{\mathrm{i}}-\theta_{\mathrm{j}}+180^{\circ} & \text { if } \theta_{\mathrm{i}}-\theta_{\mathrm{j}}<-90^{\circ} \\ \theta_{\mathrm{i}}-\theta_{\mathrm{j}}-180^{\circ} & \text { if } \theta_{\mathrm{i}}-\theta_{\mathrm{j}} \geq 90^{\circ}\end{cases}
$$

and $\Delta a b s\left(\theta_{\mathrm{i}}, \theta_{\mathrm{j}}\right) \in\left[0^{\circ}, 90^{\circ}\right]$ returns the difference between $\theta_{\mathrm{i}}, \theta_{\mathrm{j}} \in\left[-90^{\circ}, 90^{\circ}\right)$ :

$$
\operatorname{\Delta abs}\left(\theta_{\mathrm{i}}, \theta_{\mathrm{j}}\right)= \begin{cases}\left|\theta_{\mathrm{i}}-\theta_{\mathrm{j}}\right| & \text { if }\left|\theta_{\mathrm{i}}-\theta_{\mathrm{j}}\right|<90^{\circ} \\ || \theta_{\mathrm{i}}-\theta_{\mathrm{j}}\left|-180^{\circ}\right| & \text { otherwise }\end{cases}
$$

$\mu$ is the weight of the orientation difference contribution, and $\operatorname{card}(\mathcal{A})$ returns the number of triplets in $\mathcal{A}$. The orientation difference evaluation is the only contribution inherited by the inexact graph matching step in [18]. Actually, only a few orientation differences are taken into account, since most of them are nondiscriminant (e.g., in the upper portion of each fingerprint, the ridge lines describe a semi-circle, causing very similar orientation differences among the distinct typologies).

The application cost of a dynamic mask $M$ to a directional image $\mathrm{D}$ is computed by determining the minimum $\operatorname{cost} C_{\mathrm{sm}}\left(\mathrm{M}_{\mathrm{T}^{*}, \mathrm{Q}^{*}}, \mathrm{D}\right)$ over all the possible steady masks $\mathrm{M}_{\mathrm{T}, \mathrm{Q}}$ :

$$
\mathrm{C}_{d m}(M, D)=C_{\mathrm{sm}}\left(\mathrm{M}_{\mathrm{T}^{*}, \mathrm{Q}^{*}}, \mathrm{D}\right)=\min _{\mathrm{T}, \mathrm{Q}}\left(C_{\mathrm{sm}}\left(\mathrm{M}_{\mathrm{T}, \mathrm{Q}}, \mathrm{D}\right)\right) .
$$

Fig. 10 shows the adaptation of the same mask to three different images of the same fingerprint.

In order to determine the application cost $C_{\mathrm{dm}}(\mathrm{M}, \mathrm{D})$ we adopt a heuristic strategy which sequentially attempts to optimize the parameters involved, according to the following steps:

1) Selection of the best global rotation-displacement $T^{*}$ by means of an exhaustive search over a range of discrete rotation-displacements of $\mathrm{M}$, fixing $\mathrm{Q}=\{(0,0)$, $(0,0), \ldots\}$. 

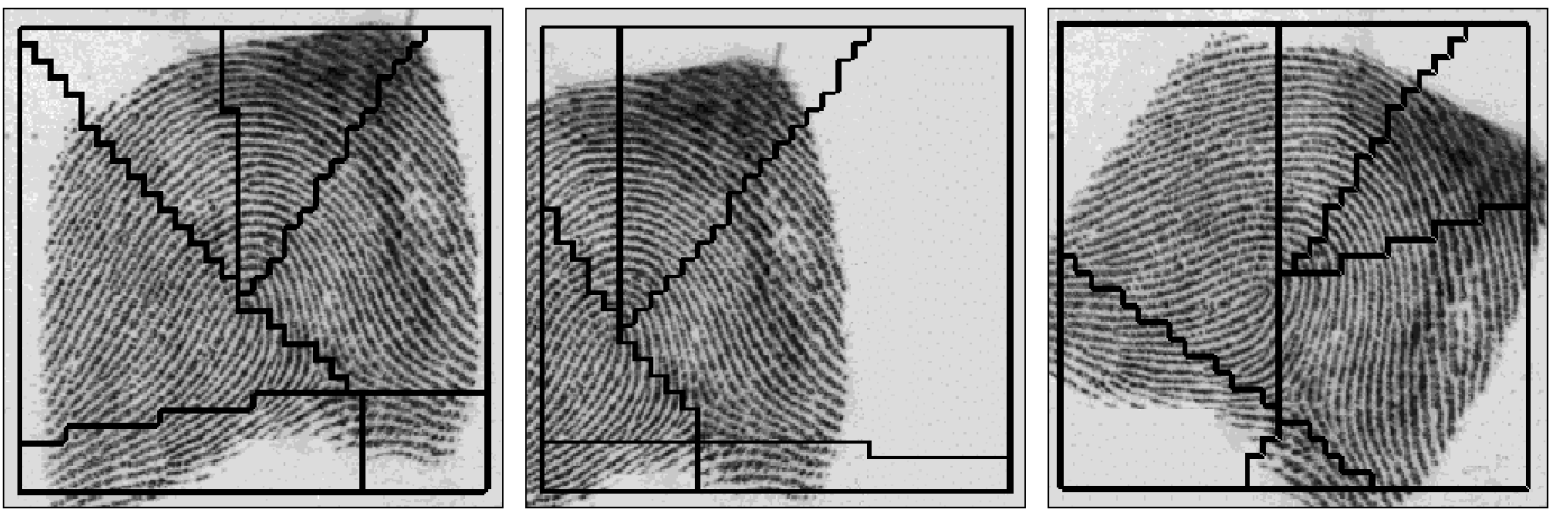

Fig. 10. Adaptation of the mask defined in Fig. 9 to three different images of the same Left Loop fingerprint.
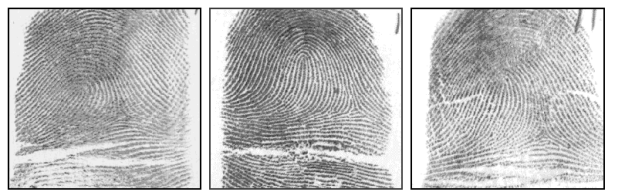

Selection of a set of representative

fingerprints belonging to one class

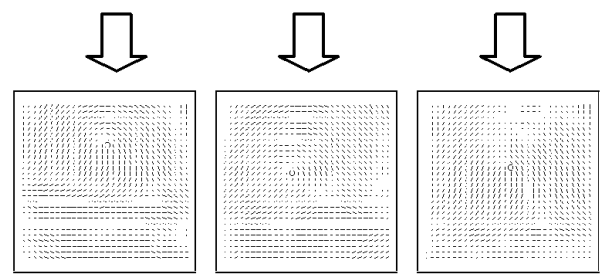

Computation and enhancement of the directional images
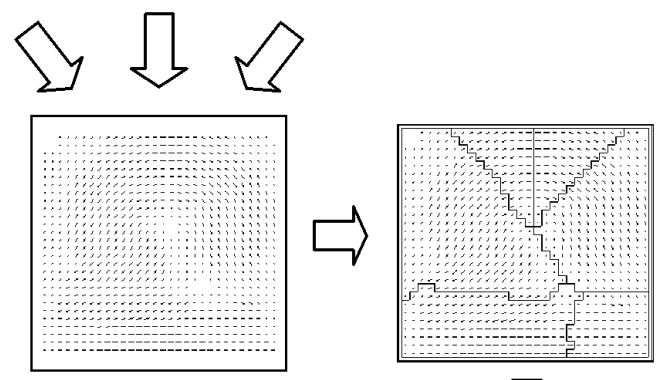

Average directional image construction and segmentation by means of the Genetic Algorithm derived from [17]

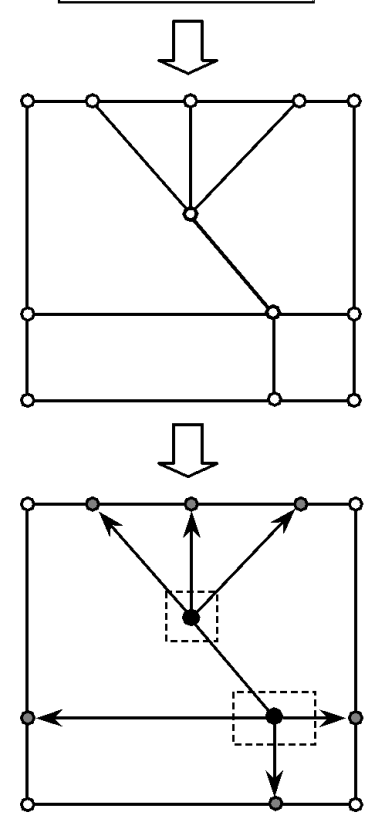

Derivation by abstraction of a vectorial mask made of vertices and segments

Definition of fixed, mobile and dependent vertices and of the mobility windows. A mobile vertex is created corresponding to each singularity. Orientation differences are derived.

Fig. 11. Prototype mask creation. The mask area is larger than the directional image to allow the border elements to be considered during the mask displacement. 


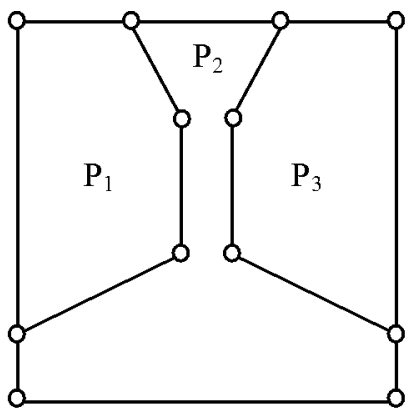

Arch

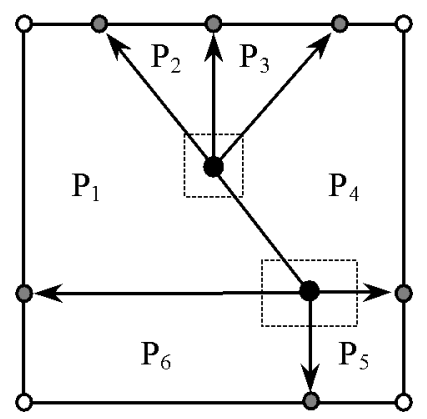

Left Loop

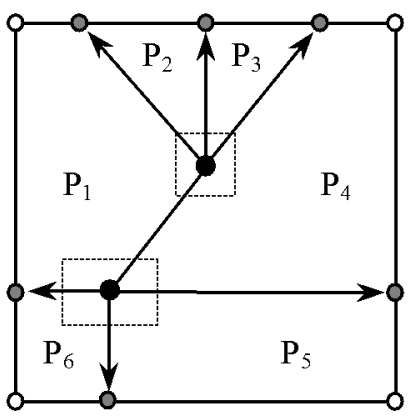

Right Loop

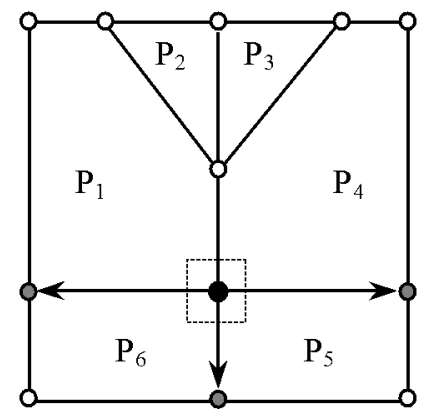

Tented Arch

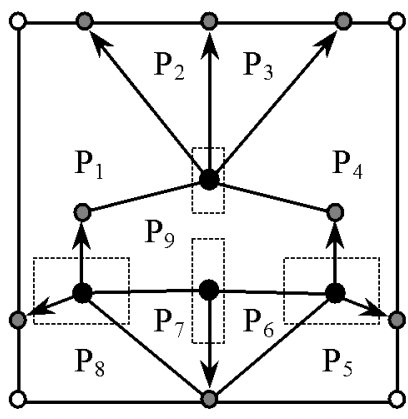

Whorl
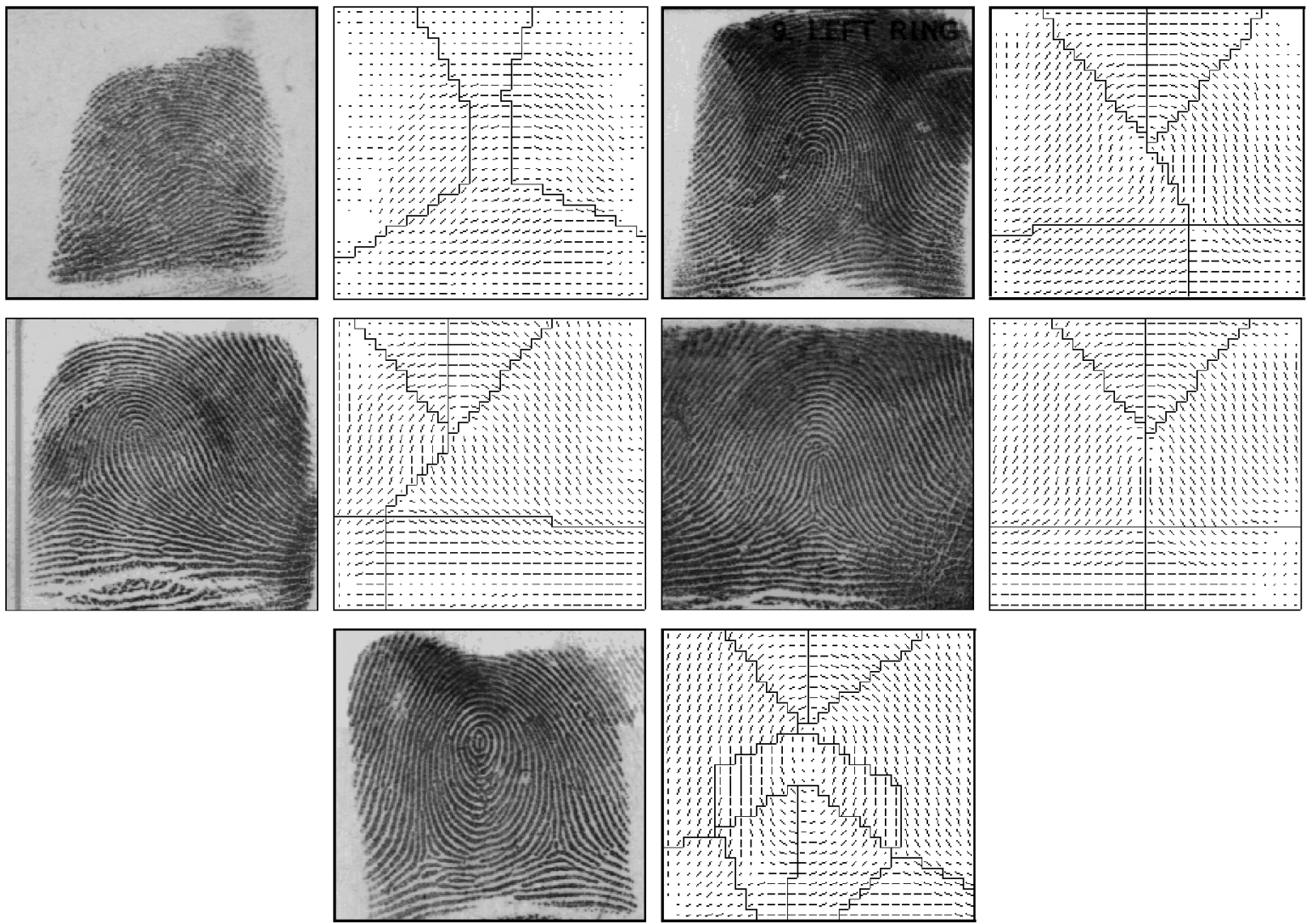

Fig. 12. The five prototype masks derived from the classes Arch, Left Loop, Right Loop, Tented Arch, and Whorl and an example of application of each mask to a fingerprint belonging to the corresponding class. The vertex positions, the mobility windows, and the dependencies on mobile vertices are graphically shown; the orientation differences are: $\mathcal{A}_{\text {Arch }}=\left\{\left(\mathrm{P}_{1}, \mathrm{P}_{2}, 37^{\circ}\right),\left(\mathrm{P}_{2}, \mathrm{P}_{3}, 37^{\circ}\right)\right\}, \mathcal{A}_{\text {Left Loop }}=\left\{\left(\mathrm{P}_{1}, \mathrm{P}_{6}, 36^{\circ}\right)\right\}, \mathcal{A}_{\mathrm{Right}}$ Loop $=$ $\left\{\left(\mathrm{P}_{4}, \mathrm{P}_{5},-36^{\circ}\right)\right\}, \mathcal{A}_{\text {Tented Arch }}=\left\{\left(\mathrm{P}_{1}, \mathrm{P}_{4},-38^{\circ}\right)\right\}, \mathcal{A}_{\text {Whorl }}=\left\{\left(\mathrm{P}_{1}, \mathrm{P}_{9},-30^{\circ}\right),\left(\mathrm{P}_{4}, \mathrm{P}_{9}, 30^{\circ}\right)\right\}$. 

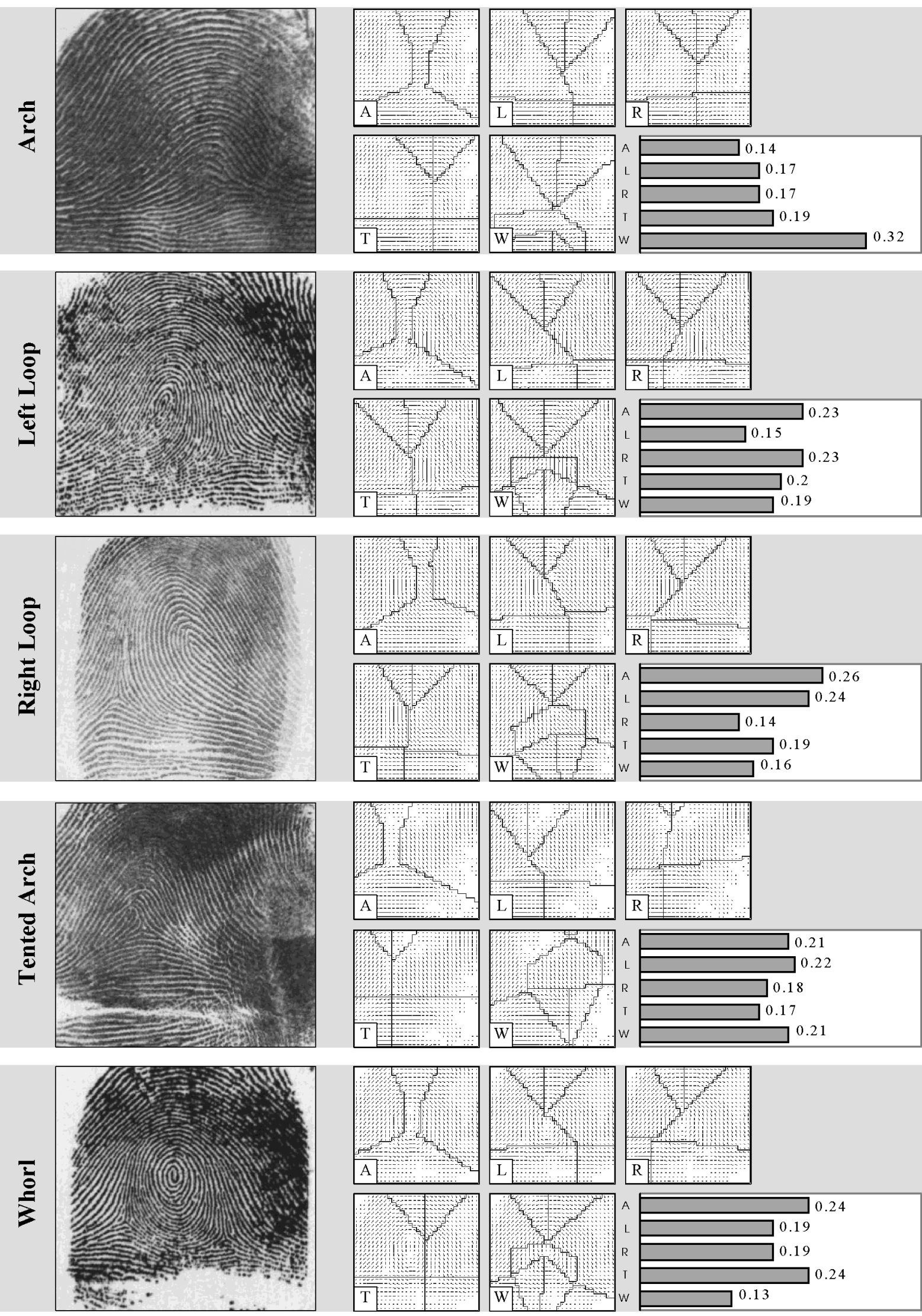

Fig. 13. The figure shows the segmentation obtained by applying the prototype masks defined in Fig. 12 to some sample fingerprints (only one example is provided for each class); the corresponding normalized feature vectors are shown on the right in the form of histograms. 
2) For each mobile vertex $\mathbf{p}_{\mathrm{i}} \in \mathrm{V}_{\mathrm{M}}$, selection of the best displacement $\left(\mathrm{d}_{\mathrm{x} \text { i, }}^{*} \mathrm{~d}_{\mathrm{y} i \mathrm{i}}^{*}\right)$ with respect to the initial position, by means of an exhaustive search over a range of discrete positions within the window $f_{\text {win }}\left(\mathbf{p}_{\mathrm{i}}\right)$, being $\mathrm{T}=\mathrm{T}^{*}$ and $\mathrm{Q}=\left\{\left(\mathrm{d}_{\mathrm{x} 1}^{*}, \mathrm{~d}_{\mathrm{y} 1}^{*}\right), \ldots,\left(\mathrm{d}_{\mathrm{x} \mathrm{i}-1}^{*}, \mathrm{~d}_{\mathrm{y} \mathrm{i}-1}^{*}\right),\left(\mathrm{d}_{\mathrm{xi}}, \mathrm{d}_{\mathrm{y} \mathrm{i}}\right), \ldots\right.$, $(0,0)\}$; the mobile vertices already considered are maintained in the optimal positions found, whereas those not yet considered are placed in the initial positions.

The sequential optimization and the discretization adopted for global mask displacement, global mask rotation, and mobile vertex positions allow the number of evaluations of the segmentation cost to be reduced, thus obtaining several computational advantages; on the other hand, the final cost is not guaranteed to be the optimum.

\subsection{Generation of a Set of Prototype Masks}

The creation of prototype masks is performed once and for all. For each possible fingerprint pattern, at least one wellfitting dynamic mask should be created. Hence, a reasonable solution is to derive the masks from the classes of one well-known classification scheme. The approach summarized in Fig. 11 has been adopted to create each dynamic mask. The set of masks used in our experimentation is reported in Fig. 12.

\subsection{Classification}

Let $\mathrm{M}_{\mathrm{i}}, \mathrm{i}=1$..s be the prototype masks and $\mathrm{D}$ the directional image to be classified; the feature vector $\mathbf{w}_{\mathrm{D}}$ resulting from the mask application is:

$$
\mathbf{w}_{\mathrm{D}}=\left[C_{\mathrm{dm}}\left(\mathrm{M}_{1}, \mathrm{D}\right), C_{\mathrm{dm}}\left(\mathrm{M}_{2}, \mathrm{D}\right), \ldots, C_{\mathrm{dm}}\left(\mathrm{M}_{\mathrm{s}}, \mathrm{D}\right)\right],
$$

where low component values denote high similarity with the corresponding prototype mask. $\mathbf{w}_{\mathrm{D}}$ can be normalized as:

$$
\begin{gathered}
\tilde{\mathbf{w}}_{\mathrm{D}}=\left[C_{\mathrm{dm}}\left(\mathrm{M}_{1}, \mathrm{D}\right) / C_{\Sigma}, C_{\mathrm{dm}}\left(\mathrm{M}_{2}, \mathrm{D}\right) / C_{\Sigma}, \ldots, C_{\mathrm{dm}}\left(\mathrm{M}_{\mathrm{s}}, \mathrm{D}\right) / C_{\Sigma}\right] \\
C_{\Sigma}=C_{\mathrm{dm}}\left(\mathrm{M}_{1}, \mathrm{D}\right)+C_{\mathrm{dm}}\left(\mathrm{M}_{2}, \mathrm{D}\right)+\ldots+C_{\mathrm{dm}}\left(\mathrm{M}_{\mathrm{s}}, \mathrm{D}\right)
\end{gathered}
$$

The normalization enables:

- working within the fixed range $[0,1]$; this makes fingerprint indexing through spatial data structures easier.

- dealing with differently contrasted images: The image contrast is related to the magnitude of the directional elements; hence, it can determine an increase or a reduction of all the costs.

If an exclusive classification is required, a neural network or a statistical classifier can be used to properly classify vectors $\widetilde{\mathbf{w}}_{\mathrm{D}}$. In a continuous approach, $\widetilde{\mathbf{w}}_{\mathrm{D}}$ itself can be used as an access key for similarity searches; in fact, it is reasonable to assume that similar directional images give analogous segmentation costs and therefore the corresponding vectors are close to each other in the sdimensional space. Fig. 13 shows the feature vectors $\tilde{\mathbf{w}}_{D}$ extracted from fingerprints belonging to different classes.

\section{FINGERPRINT RETRIEVAL}

Several strategies for fingerprint retrieval can be defined according to the application requirements: accuracy/efficiency needed, the kind of minutiae matching algorithm used, the presence of a human supervisor, etc. For example, Senior proposed in [23] a retrieval technique based on exclusive classification, where more than one class are searched in case of classification uncertainty; the trade-off between retrieval efficiency against error rate can be tuned according to the user requirements. In order to evaluate the efficiency of continuous vs. exclusive classification for latent fingerprint retrieval, two different methodologies were proposed in [16]. The two methodologies (named A and B) are applicable to both the classification approaches, thus obtaining four different strategies (respectively, AE, AC, BE, BC ). In any case, the classification enables reducing the number of fingerprints which have to be considered for minutiae matching; the matching step produces a small list of candidate fingerprints and the final decision of real correspondence is taken by a human expert who analyzes the candidate fingerprints only.

\subsection{Methodology A}

Methodology A assumes an error-free classification, so the search is restricted to the database fingerprints resembling analogous classification characteristics. The strategy AE can be implemented by searching the whole corresponding class of the latent fingerprint; the strategy AC, by searching among those fingerprints which are less far from the feature vector $\mathbf{w}$ of the latent fingerprint than a fixed tolerance $\rho$. The average portion of database considered and the average retrieval error can be formally stated as:

AE : Given an exclusive classification scheme with s classes, let $\mathrm{P}_{\mathrm{d}}(\mathrm{i}), \mathrm{i}=1$..s be the probability that a fingerprint belongs to the class $i$ according to the database class distribution and let $\mathrm{P}_{\mathrm{c}}(\mathrm{i}), \mathrm{i}=1$..s be the probability that a latent fingerprint is assigned to the class $i$; then, the average portion of database considered $\mathrm{C}(\mathrm{AE})$ is:

$$
\mathrm{C}(\mathrm{AE})=\sum_{\mathrm{i}=1}^{\mathrm{s}} \mathrm{P}_{\mathrm{c}}(\mathrm{i}) \cdot \mathrm{P}_{\mathrm{d}}(\mathrm{i})
$$

where $P_{d}(i)$ represents the database fraction involved in the retrieval of a fingerprint of class $i$ and $P_{c}(i)$ is the weighting factor representing the probability to classify a latent fingerprint as $i$. Let $P_{d \mid c}(j \mid i)$ be the conditional probability that a database fingerprint, corresponding to a latent fingerprint classified as $i$, has been classified $j$ in the database; then, the average retrieval error $\mathrm{E}(\mathrm{AE})$ can be calculated by weighting the average retrieval errors of the single classes by the probabilities $\mathrm{P}_{\mathrm{c}}(\mathrm{i})$ :

$$
\begin{aligned}
& E(A E)=\sum_{i=1}^{s} P_{c}(i) \cdot E_{i}(A E) \\
& E_{i}(A E)=\sum_{j=1, j \neq i}^{s} P_{d \mid c}(j \mid i)
\end{aligned}
$$

Ac : Given a fixed tolerance $\rho$, the average portion of database considered $C_{\rho}(A C)$ is determined by the average number of fingerprints inside the hyper-sphere with ra- 
dius $\rho$ centered in the latent fingerprint. The average retrieval error $\mathrm{E}_{\rho}(\mathrm{AC})$ is determined by the average number of missed retrievals inside the search area.

\subsection{Methodology B}

Methodology B allows for misclassification to be taken into account; to this aim, the search is carried out incrementally over the whole database, avoiding any possible retrieval error. This methodology requires the search to be terminated when a human expert finds a real correspondence between the latent fingerprint and a database fingerprint that has already been considered. If the latent fingerprint has no correspondence in the database the search is always extended to the whole database. The strategy BE can be implemented by starting the search from the latent fingerprint class, and incrementally extending it to the other classes; the strategy BC can be carried out by processing fingerprints according to their distance from the latent fingerprint feature vector $\mathbf{w}$.

BE : Let $q^{i}=\left\langle q_{1}{ }^{i}, q_{2}, \ldots, q_{s}^{i}\right\rangle$ be a permutation defining a class sequence for the retrieval of an i fingerprint. On average, half a class has to be scanned to find the fingerprint corresponding to the latent one, if it exists; and the whole class has to be scanned, otherwise. Therefore, when a correspondence exists in the database, the average portion of database considered $\mathrm{C}(\mathrm{BE})$ is:

$$
\begin{gathered}
C(B E)=\sum_{i=1}^{s} P_{c}(i) \cdot C_{i}(B E) \\
C_{i}(B E)=\sum_{j=1}^{s}\left(P_{d \mid c}\left(q_{j}^{i} \mid i\right) \cdot\left[\frac{1}{2} P_{d}\left(q_{j}^{i}\right)+\sum_{k=1}^{j-1} P_{d}\left(q_{k}{ }^{i}\right)\right]\right),
\end{gathered}
$$

where the term between the square brackets represents the average portion of database considered when the corresponding fingerprint of an i latent fingerprint belongs to the $j$ th class $\left(\mathrm{q}_{\mathrm{j}}^{\mathrm{i}}\right)$; in fact, this term includes the scan of all the fingerprints belonging to the classes which precede $q_{j}^{i}$ in $q^{i}$ and the scan of half the fingerprints belonging to $\mathrm{q}_{\mathrm{j}}^{\mathrm{i}}$. The optimum sequence $\mathrm{q}^{*^{\mathrm{i}}}$ can be determined according to the following ordering rule. Let $a$ and $b$ be two adjacent classes in the optimum sequence $q^{* i}$, then a precedes $b$ if and only if $P_{d \mid c}(b \mid i) \cdot P_{d}(a)<$ $P_{d \mid c}(a \mid i) \cdot P_{d}(b)$; in fact, it can be simply proven that, by exchanging the order of $a$ and $b$, the corresponding $\mathrm{C}_{\mathrm{i}}(\mathrm{BE})$ values differ only for the above-considered terms. Furthermore, the transitive property for the class precedence rule can be proved.

BC: The average portion of database considered $\mathrm{C}(\mathrm{BC})$ is determined by the average number of fingerprints inside the hyper-sphere centered in $\mathbf{w}$ and with radius $\rho$ given by the distance between $\mathbf{w}$ and $\mathbf{v}$, where $\mathbf{v}$ is the feature vector relative to the database fingerprint corresponding to the searched one.

\section{EXPERIMENTAL RESULtS}

This section reports some experimental results obtained by the dynamic mask method and compares it with other classification approaches, using two fingerprint databases commonly adopted by the scientific community. In the following, we denote with MASK the dynamic mask method introduced here, with LUMINI the continuous classification approach described in [16], with PCASYS the exclusive approach by NIST [4], and with KARU the exclusive approach by Karu and Jain [13].

\subsection{Databases}

The experimentation has been performed on NIST Special Database 4 (Db4) [27] and NIST Special Database 14 (Db14) [28]. Both databases consist of 256 gray-level images; two different fingerprint instances $(\mathrm{F}=$ First, $\mathrm{S}=$ Second $)$ are present for each finger. Each fingerprint was manually analyzed by a human expert and assigned to one of the following five classes: Arch (A), Left Loop (L), Right Loop (R), Tented Arch (T), and Whorl (W). Actually, some ambiguous fingerprints have an additional reference to one or more classes (cross-referenced fingerprints).

- Db4 contains 2,000 fingerprint pairs, uniformly distributed in the five classes; in order to resemble a real distribution $(\mathrm{A}=3.7$ percent, $\mathrm{T}=2.9$ percent, $\mathrm{L}=33.8$ percent, $\mathrm{R}=31.7$ percent, $\mathrm{W}=27.9$ percent), we reduced the cardinality of the less frequent classes, obtaining 1,204 pairs (the first fingerprints from each class have been chosen according to the right proportion).

- Db14 contains 27,000 fingerprint pairs randomly taken, thus approximating the real fingerprint distribution; only the last 2,700 fingerprint pairs have been employed in our simulation, since these constitute the test set used in [4].

The first 2,000 fingerprints of Db14 have been used as "training set" to derive the set of prototype masks and to optimize the parameters of our method. Actually, since the mask construction concerns macroscopic features of the fingerprints, it can be verified that prototypes created starting from different training sets (provided they are sufficiently large) are almost identical. The parameter values used for the tests are: $\sigma=9.6, \lambda=112, \mu=1.6, C_{0}=10$; the range of global mask displacements is $[-8,8]$ for both $\mathrm{x}$ and $\mathrm{y}$ axes, with discrete steps of two blocks; the range of global mask rotation is $\left[-20^{\circ}, 20^{\circ}\right]$ with steps of $5^{\circ}$.

\subsection{Continuous Classification for Fingerprint Retrieval}

In order to compare the performance of the two continuous approaches MASK and LUMINI, some fingerprint retrieval operations have been simulated, according to strategies AC and BC described in Section 4, by indexing the fingerprint instances $\mathrm{F}$ through their feature vectors and by searching the corresponding instances $\mathrm{S}$. Moreover, the retrieval results of PCASYS are evaluated in the context of strategies AE and BE, and compared with those given by the continuous approaches.

\subsubsection{Methodology A}

In methodology $\mathrm{A}$, continuous classification allows defining the reliability level a priori by tuning $\rho$ (i.e., increasing the time spent for retrieval to decrease the average retrieval error). Fig. 14 reports the results produced by MASK as to the average retrieval error $\mathrm{E}_{\rho}(\mathrm{AC})$ and the average portion of database considered $\mathrm{C}_{\rho}(\mathrm{AC})$. 


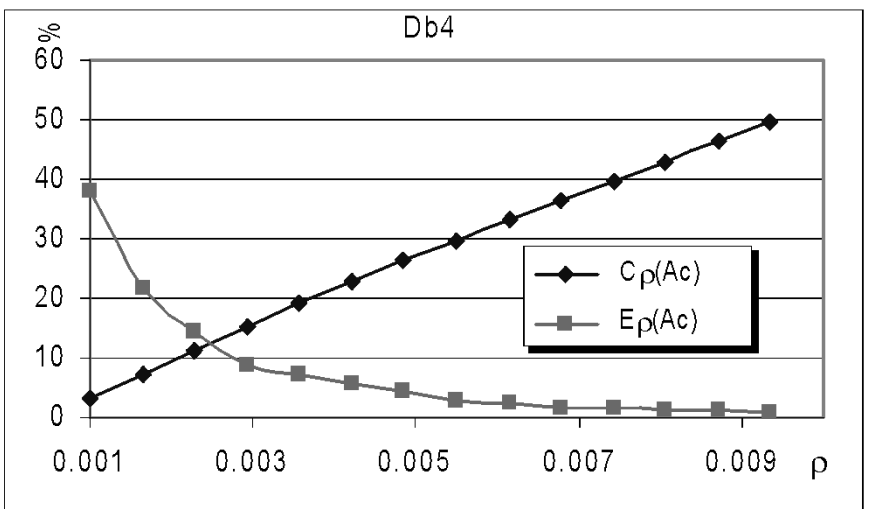

(a)

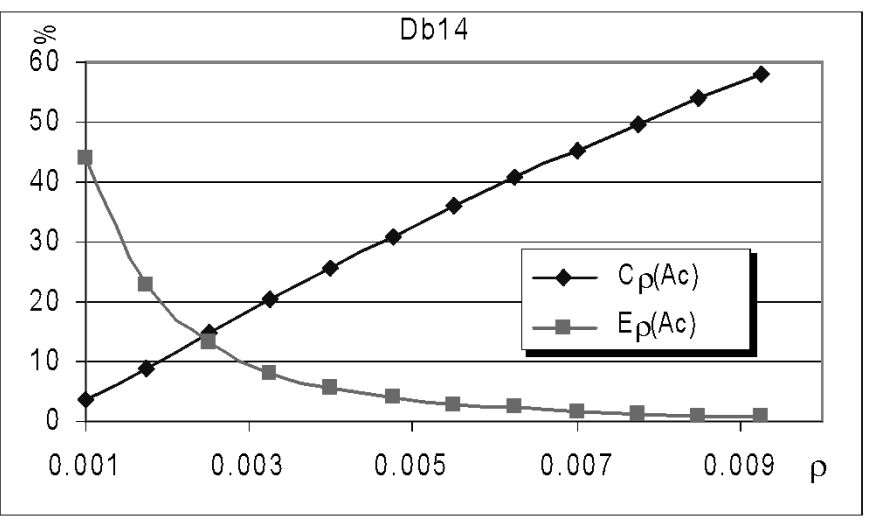

(b)

Fig. 14. MASK results over Db4 (a) and Db14 (b); the average portion of database considered $C_{\rho}(\mathrm{Ac})$ and the average retrieval error $\mathrm{E}_{\rho}(\mathrm{Ac})$ are plotted as a function of $\rho$.

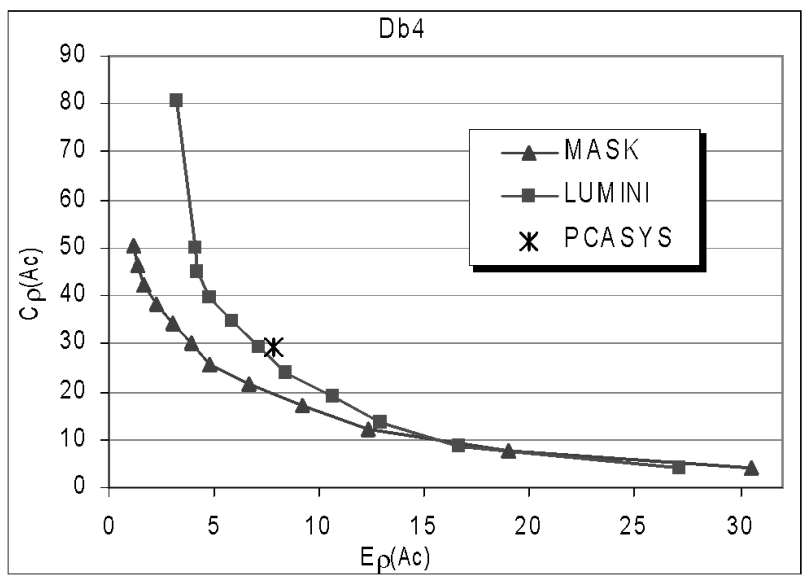

(a)

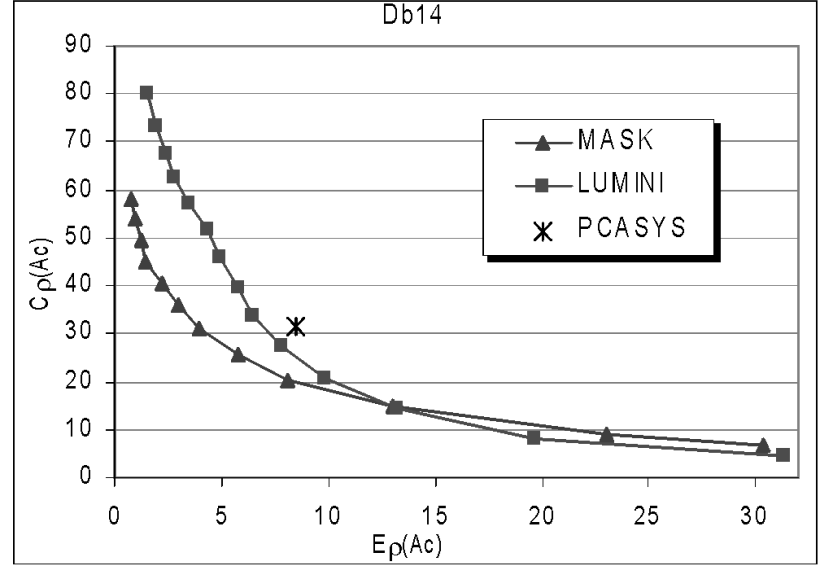

(b)

Fig. 15. Trade-off $C_{\rho}(A C) / E_{\rho}(A C)$ varying $\rho$ for the two continuous approaches MASK and LUMINI. The point $X$ denotes $C(A E) / E(A E)$ for the exclusive classification approach PCASYS.

TABLE 1

Strategy AC: COMPARISON BETWEEN LUMINI AND MASK

\begin{tabular}{|c|c|c|c|c|}
\hline \multirow{2}{*}{$\mathrm{E}_{\rho}(\mathrm{AC})$} & \multicolumn{4}{|c|}{$\mathrm{C}_{\rho}(\mathrm{AC})$} \\
\cline { 2 - 5 } & \multicolumn{2}{|c|}{$\mathrm{Db} 4$} & \multicolumn{2}{c|}{$\mathrm{Db} 14$} \\
\cline { 2 - 5 } & LUMINI & MASK & LUMINI & MASK \\
\hline $2 \%$ & $77.5 \%$ & $39.4 \%$ & $73.1 \%$ & $41.5 \%$ \\
\hline $4 \%$ & $50.0 \%$ & $29.4 \%$ & $52.5 \%$ & $30.6 \%$ \\
\hline $6 \%$ & $34.4 \%$ & $22.0 \%$ & $35.4 \%$ & $24.5 \%$ \\
\hline $8 \%$ & $26.2 \%$ & $19.6 \%$ & $27.6 \%$ & $20.9 \%$ \\
\hline $10 \%$ & $20.1 \%$ & $14.3 \%$ & $20.3 \%$ & $17.8 \%$ \\
\hline $14 \%$ & $11.6 \%$ & $10.8 \%$ & $13.4 \%$ & $13.8 \%$ \\
\hline
\end{tabular}

The graphs in Fig. 15 summarize MASK performance by plotting each pair $\mathrm{E}_{\rho}(\mathrm{AC}), \mathrm{C}_{\rho}(\mathrm{AC})$ relative to the same $\rho$ as a single point; LUMINI performance and PCASYS performance are also reported. MASK performance is considerably better than LUMINI at low error rate (see Table 1); as the error increases, the difference between the two methods progressively disappears and, for error rates beyond 14 percent, the two methods behave similarly.
In order to compare the two continuous approaches with PCASYS, which determines just one operating point in the graphs in Fig. 15, the abscissa or the ordinate value should be fixed. Table 2 reports the average errors $\mathrm{E}(\mathrm{AE}), \mathrm{E}_{\rho}(\mathrm{AC})$ obtained by fixing the ordinate value $C_{\rho}(A C)=C(A E)=$ $\mathrm{C}(\mathrm{A})$. It should be noted that MASK error is considerably lower than the others. 
TABLE 2

Comparison Among PCASYS, LUMINI, ANd MASK FIXING the Average Portion of DatABASE ReAD

\begin{tabular}{|l|c|c|c|}
\hline \multicolumn{5}{|c|}{ Db4 } \\
\hline \multirow{2}{*}{$\mathrm{C}(\mathrm{A})=29.12 \%$} & PCASYS & LUMINI & MASK \\
\cline { 2 - 4 } & $\mathrm{E}(\mathrm{AE})=7.81 \%$ & $\mathrm{E}_{\rho}(\mathrm{AC})=7.13 \%$ & $\mathrm{E}_{\rho}(\mathrm{AC})=3.99 \%$ \\
\hline $\mathrm{Db} 14$ & PCASYS & LUMINI & MASK \\
\hline \multirow{2}{*}{$\mathrm{C}(\mathrm{A})=31.29 \%$} & $\mathrm{E}(\mathrm{AE})=8.49 \%$ & $\mathrm{E}_{\rho}(\mathrm{AC})=6.75 \%$ & $\mathrm{E}_{\rho}(\mathrm{AC})=3.93 \%$ \\
\cline { 2 - 4 }
\end{tabular}

TABLE 3

Comparison Between the Average Percentages of Database Searched (Methodology B)

\begin{tabular}{|c|c|c|c|c|c|}
\hline \multicolumn{3}{|c|}{ Db4 } & \multicolumn{3}{c|}{ Db 14} \\
\hline PCASYS & LUMINI & MASK & PCASYS & LUMINI & MASK \\
\hline $\mathrm{C}(\mathrm{BE})=17.16 \%$ & $\mathrm{C}(\mathrm{BC})=6.90 \%$ & $\mathrm{C}(\mathrm{BC})=5.22 \%$ & $\mathrm{C}(\mathrm{BE})=18.91 \%$ & $\mathrm{C}(\mathrm{BC})=7.11 \%$ & $\mathrm{C}(\mathrm{BC})=6.41 \%$ \\
\hline
\end{tabular}

\subsubsection{Methodology B}

Table 3 reports the average portions of database considered for the retrieval of a given fingerprint. Two slightly different optimum sequences have been adopted to determine $\mathrm{C}(\mathrm{BE})$ for the method PCASYS on Db4 and Db14. In fact, the marginal and conditional probabilities have been derived from the experimental confusion matrices, which were different in the two cases.

Db4: $\mathbf{q}^{* \mathrm{~A}}=<\mathrm{A}, \mathrm{T}, \mathrm{R}, \mathrm{L}, \mathrm{W}>\mathrm{q}^{*} \mathrm{~L}=<\mathrm{L}, \mathrm{T}, \mathrm{A}, \mathrm{W}, \mathrm{R}>$, $\mathrm{q}^{* \mathrm{R}}=<\mathrm{R}, \mathrm{A}, \mathrm{T}, \mathrm{W}, \mathrm{L}>, \mathrm{q}^{*} \mathrm{~T}=<\mathrm{T}, \mathrm{A}, \mathrm{L}, \mathrm{R}, \mathrm{W}>, \mathrm{q}^{*} \mathrm{~W}=<\mathrm{W}, \mathrm{L}, \mathrm{R}, \mathrm{T}, \mathrm{A}>$

Db14: $\mathbf{q}^{* A}=<A, T, R, L, W>, q^{*}=<L, A, T, W, R>$, $\mathrm{q}^{* \mathrm{R}}=<\mathrm{R}, \mathrm{T}, \mathrm{A}, \mathrm{W}, \mathrm{L}>, \mathrm{q}^{*} \mathrm{~T}=<\mathrm{T}, \mathrm{A}, \mathrm{R}, \mathrm{L}, \mathrm{W}>, \mathrm{q}^{*} \mathrm{~W}=<\mathrm{W}, \mathrm{L}, \mathrm{R}, \mathrm{T}, \mathrm{A}>$

For LUMINI and MASK, C(BC) has been experimentally determined by counting, for each instance $S$, how many instances $\mathrm{F}$ were closer to $\mathrm{S}$ than its corresponding fingerprint, and by averaging the results. Table 3 shows that MASK performs slightly better than LUMINI and, especially, that both the continuous approaches outperform the exclusive approach PCASYS.

\subsection{Exclusive Classification}

Although MASK was conceived for continuous classification, its prototype masks derive from the classes $\{\mathrm{A}, \mathrm{L}, \mathrm{R}, \mathrm{T}, \mathrm{W}\}$ and, therefore, it is reasonable to expect good performance even for exclusive classification. On the other hand, the development of a neural or statistical classifier capable of improving exclusive classification performance is out of the aim of this work and a simple minimum cost criterion has just been adopted here.

TABLE 4

EXCLUSIVE ClassificATION PERCENTAge ERRORS

\begin{tabular}{|l|c|c|}
\cline { 2 - 3 } \multicolumn{1}{c|}{} & $\mathrm{Db} 4$ & $\mathrm{Db} 14$ \\
\hline MASK & $12.9 \%$ & $14.3 \%$ \\
\hline PCASYS (aux) & $9.3 \%$ & $7.7 \%$ \\
\hline PCASYS (no aux) & $11.6 \%$ & $9.6 \%$ \\
\hline KARU & $11.9 \%$ & --- \\
\hline
\end{tabular}

Exclusive classification has been performed both on $\mathrm{Db} 4$ (1,204 fingerprints) and Db14 (2,700 fingerprints), with no rejection. In case of cross-referenced fingerprints, only the "first" class has been considered. A comparison with PCASYS is provided for Db4 and Db14 and with KARU for Db4. Table 4 summarizes the percentage errors of the methods considered. Actually, KARU performance, as reported in [13], has been calculated under slightly different conditions: In fact, the authors initially computed the percentage errors associated to the five classes over the whole database and then weighed them according to the class distribution. As to PCASYS, (aux) denotes the use of the pseudoridge tracer auxiliary module.

Both PCASYS and KARU give better results than MASK (even if the performance is near the same for $\mathrm{Db} 4$ ); on the other hand, it is worth noting that MASK does not use any heuristic rule or additional criteria to improve class separation in case of uncertainty, since the continuous classification itself provides a solution to this problem.

\subsection{Robustness}

Some experiments have been carried out to evaluate MASK robustness in case of relevant disturbances (i.e., which usually affect latent fingerprints). Table 5 shows the results obtained by applying MASK and PCASYS to some fingerprint images taken from $\mathrm{Db} 4$ (see Fig. 16) and submitted to: rotations, partial deletion, and artificial noise addition. For each image in Fig. 16, Table 5 reports the kind of perturbation, the real class, and the classification output given by the two methods; the confidence score produced by PCASYS and the minimum cost obtained by MASK are also provided (within parentheses).

Generally, MASK exhibits a more robust behavior than PCASYS. In fact, PCASYS is based on the dimensionality reduction via the Karhunen-Loève transform, whose accuracy strictly relies on a correct pattern alignment which is not always assured by the initial processing steps; moreover, the KL transform is very sensitive to partial deletion and corruption of the singularities since these regions encode large sources of variance. On the contrary, MASK involves a global optimization process which generally 
TABLE 5

CLASSIFICATION OF SOME FINGERPRINT IMAGES SUBMITTED to ARTIFICIAL PERTURBATIONS

\begin{tabular}{|c|l|l|l|l|}
\hline Image & Perturbation & Class & PCASYS & MASK \\
\hline 1 & Rotation & Left & Whorl $(73 \%)$ & Left $(0.11)$ \\
\hline 2 & Rotation & Arch & Right $(48 \%)$ & Arch $(0.15)$ \\
\hline 3 & Partial & Whorl & Left $(78 \%)$ & Whorl $(0.14)$ \\
\hline 4 & Partial & Whorl & Whorl $(90 \%)$ & Whorl $(0.15)$ \\
\hline 5 & Partial & Whorl & Whorl $(71 \%)$ & Whorl $(0.16)$ \\
\hline 6 & Partial & Right & Whorl $(42 \%)$ & Right $(0.15)$ \\
\hline 7 & Noise & Right & Right $(100 \%)$ & Right $(0.15)$ \\
\hline 8 & Noise & Whorl & Left $(80 \%)$ & Whorl $(0.15)$ \\
\hline 9 & Noise & Whorl & Right $(48 \%)$ & Whorl $(0.16)$ \\
\hline 10 & Noise & Whorl & Left $(50 \%)$ & Whorl $(0.16)$ \\
\hline
\end{tabular}

TABLE 6

Strategy BC: C(BC) as a Function of the Rejection Percentage

\begin{tabular}{|c|c|c|}
\hline \% rejection & $\mathbf{C}(\mathrm{BC})$ on Db4 & $\mathbf{C}(\mathrm{BC})$ on Db14 \\
\hline $0 \%$ & $5.22 \%$ & $6.41 \%$ \\
\hline $5 \%$ & $4.46 \%$ & $5.79 \%$ \\
\hline $10 \%$ & $4.18 \%$ & $5.41 \%$ \\
\hline $20 \%$ & $3.58 \%$ & $5.07 \%$ \\
\hline
\end{tabular}

TABLE 7

ERROR ANALYSIS OVER THE 120 WORST MATED FINGERPRINT PAIRS

\begin{tabular}{|l|c|c|c|c|c|}
\hline & I & II & III & IV & V \\
\hline \# fingerprints & 46 & 9 & 14 & 22 & 29 \\
\hline
\end{tabular}

proved to be very tolerant with respect to the abovementioned perturbations.

\subsection{Rejection and Error Analysis in MASK}

The following simple rejection criterion, based on the overall quality of the directional image, can be adopted to reject fingerprints in the case of high uncertainty.

Let $R=\left\{R_{s}\right\}$ be the partitioning of the directional image $D$ in a single region $R_{s}$; then, a quality measure $Q$ is given by the variance $\operatorname{Var}\left(\mathrm{R}_{\mathrm{s}}\right)$ (see the Appendix): In fact, $\operatorname{Var}\left(\mathrm{R}_{\mathrm{s}}\right)$ measures the directional element variance within the whole directional image, weighing each element by its magnitude. Since the magnitudes of elements within a noisy fingerprint are always low and the element directions corresponding to low-contrasted images are usually "flat," in both cases, $Q=\operatorname{Var}\left(\mathrm{R}_{\mathrm{s}}\right)$ returns low values (see Fig. 17). Therefore, the rejection criterion simply consists in rejecting fingerprints with $Q$ lower than a fixed threshold.

As far as strategy AC is concerned, the two graphs in Fig. 18 show the average retrieval error $\mathrm{E}_{\rho}(\mathrm{AC})$ as a function of the rejected fingerprint percentage, fixing the portions of database searched $C_{\rho}(A C)$.
As to strategy $\mathrm{BC}$, Table 6 reports $\mathrm{C}(\mathrm{BC})$ for different rejection percentages.

Finally, we analyzed the 120 worst mated pairs from Db4 (i.e., whose $\mathrm{F}$ and $\mathrm{S}$ fingerprints were the least similar to each other according to MASK) in order to figure out the main error causes:

1) poor quality fingerprints discarded by the rejection criterion (10 percent rejection),

2) other poor quality fingerprints not rejected,

3) fingerprints with one or more written words,

4) fingerprints with one or more lines,

5) other.

Each of the 120 pairs has been assigned to one of the above categories; precedence has been given to the category with the lowest index (e.g., a fingerprint with both words and lines is assigned to Category 3). Fig. 19 shows some fingerprints assigned to Categories 3 and 4 which appear in the NIST databases, since the corresponding images were scanned from hand-annotated FBI cards. Table 7 summarizes our error analysis: only a few low-quality fingerprints have not been rejected and most of the errors are caused by written words and/or lines. No ad hoc rejection rules have 

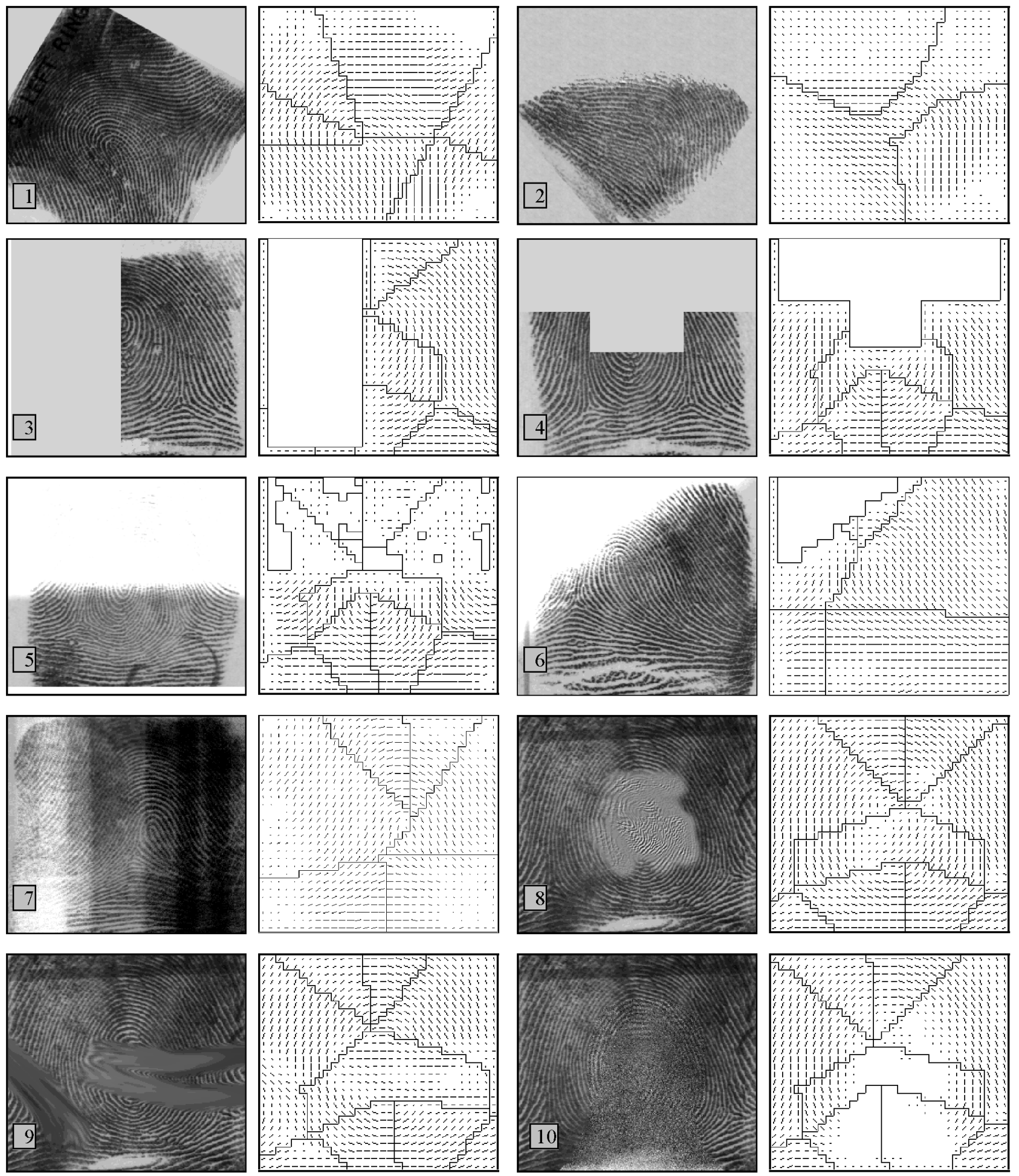

Fig. 16. Fingerprints manually altered by: rotation (no. 1, no. 2), partial deletion (no. 3, no. 4, no. 5, no. 6), and noise addition (no. 7, no. 8, no. 9, no. 10). The segmentation obtained by the lowest cost mask is reported on the right side of each image; in all cases, the classification result produced by MASK is correct.

been developed to deal with Categories 3 and 4 because these kind of perturbations are specific to the databases used.

\section{Conclusions}

In this work, a new structural approach to fingerprint classification is introduced. As in the relational graph method
[18], directional image partitioning is performed to capture the conspicuous fingerprint macro-features. Dynamic masks have been defined as a powerful instrument for a robust segmentation.

The experimental results prove the accuracy and robustness of the new method and the comparison with other 

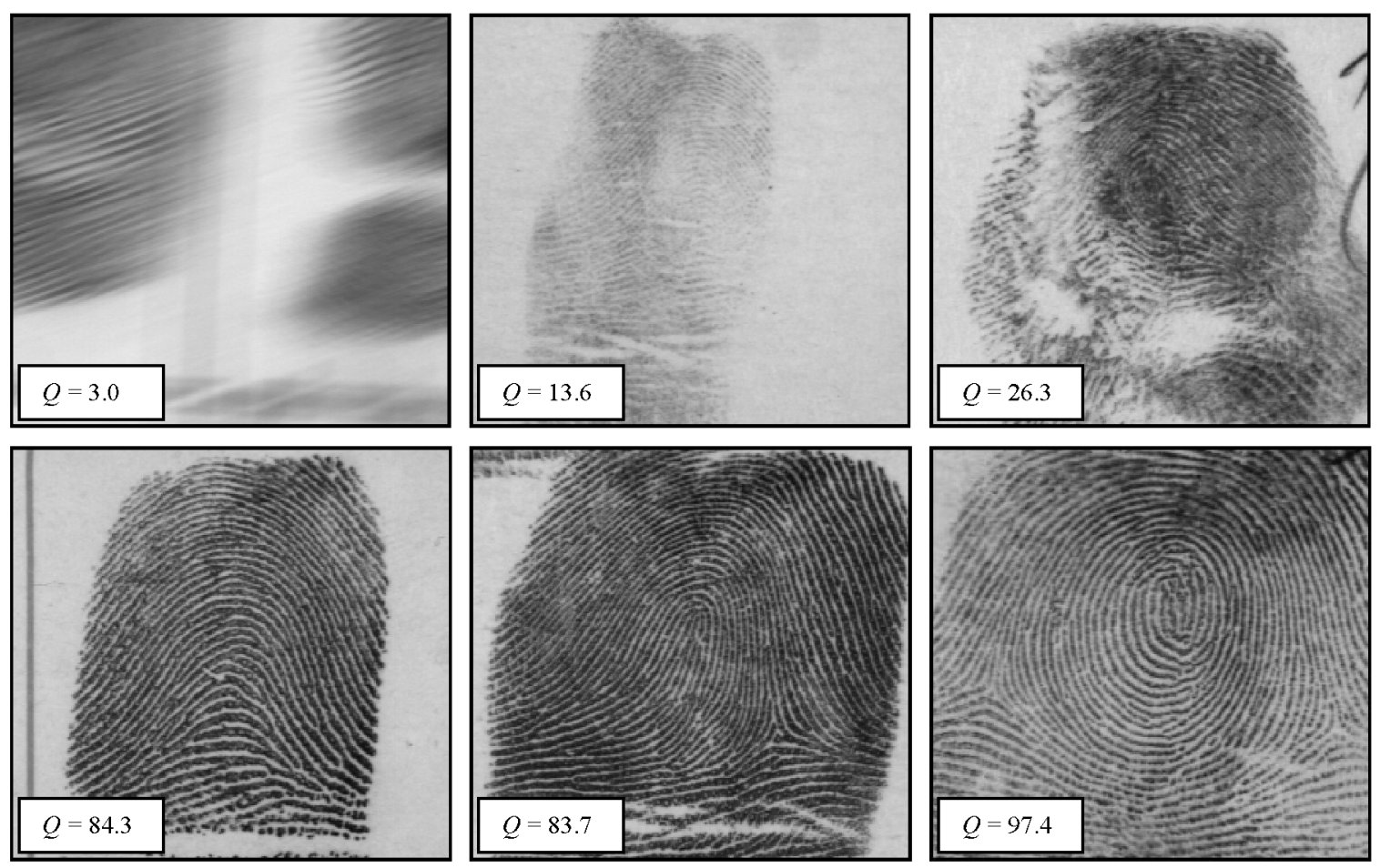

Fig. 17. Low quality (top row) and high quality (bottom row) images from Db4.
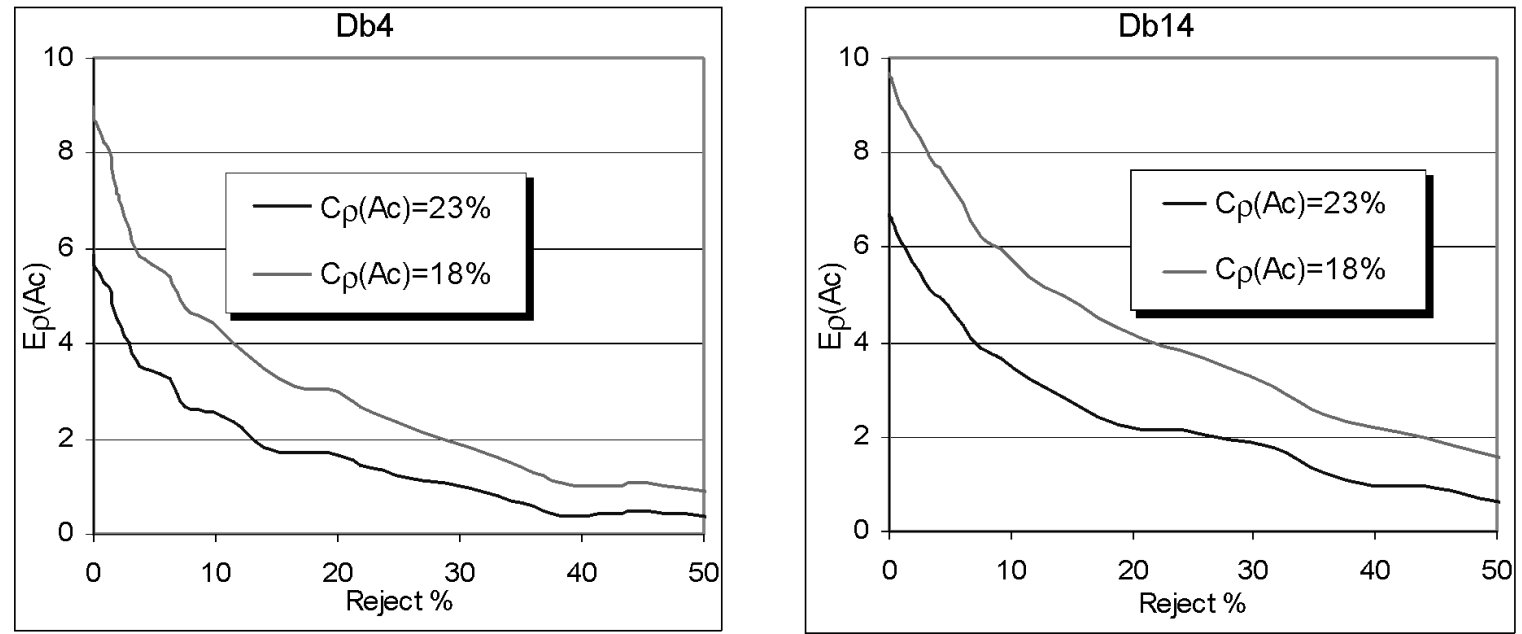

Fig. 18. Strategy $A C$ : The average error $E_{\rho}(A C)$ as a function of the rejection percentage in $D b 4$ and $D b 14$; the two curves refer to $C_{\rho}(A C)=18$ percent and $C_{\rho}(A C)=23$ percent.

techniques demonstrates its superiority for the continuous classification task. We would like to remark that the aim of our work is not to prove the superiority of the continuous classification approaches with respect to the exclusive ones. In fact, continuous classification does not enable to accomplish some tasks to be carried out, such as fingerprint labeling according to a given classification scheme. Nevertheless, if we classify fingerprints only for improving the retrieval efficiency and we operate under the hypotheses involved by the retrieval methodologies A and B, MASK approach performs better than exclusive techniques.

All the simulations have been carried out on a PC Pentium $^{\text {TM }} 200 \mathrm{MHz}$. Table 8 reports the average time neces- sary for the main processing steps involved in a fingerprint classification with the MASK approach.

Future work will be dedicated to the definition and experimentation of a new set of prototype masks not necessarily derived from the common fingerprint classes and to the definition of other rejection criteria.

\section{APPENDIX}

\section{Variance of a Region}

Given a directional image $D$ and a segmentation $R=\left\{R_{1}, R_{2}\right.$, $\ldots R_{s}$ of $D$, the variance $\operatorname{Var}\left(R_{i}\right)$ of the region $R_{i}$ containing $n_{i}$ directional elements $\mathbf{v}=\left[\mathrm{v}_{\mathrm{x}}, \mathrm{v}_{\mathrm{y}}\right]$ is defined as: 

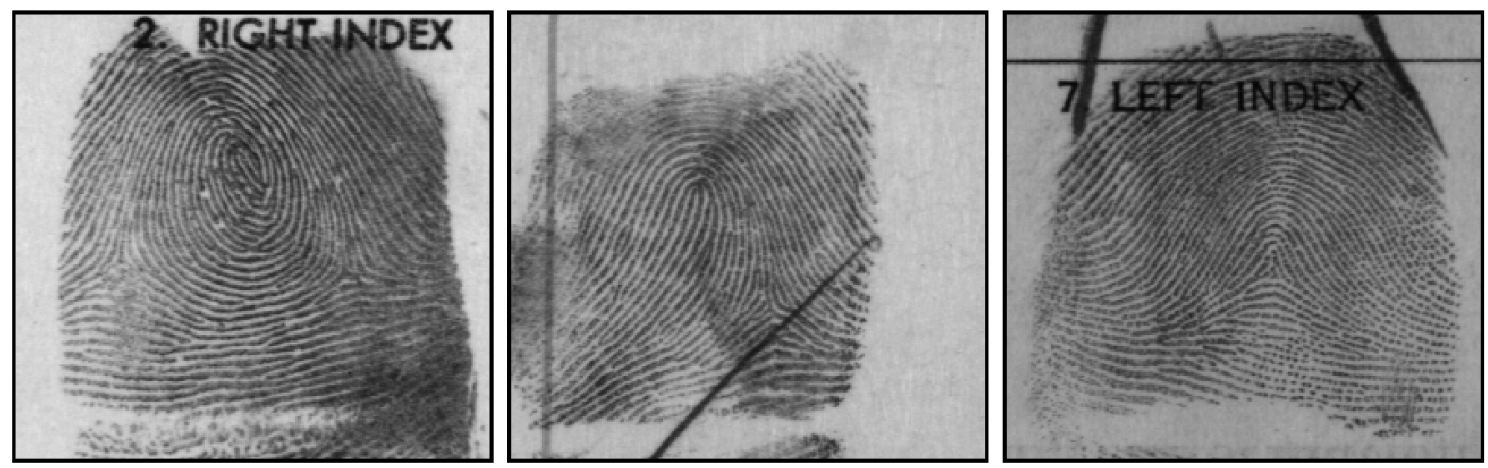

Fig. 19. Fingerprint images with written words and/or lines.

TABLE 8

Average Time Spent for the Main Processing Steps

\begin{tabular}{|l|c|}
\hline \multicolumn{1}{|c|}{ Step } & Time (sec.) \\
\hline Fingerprint image enhancement & 2.35 \\
\hline Directional image computation and enhancement & 0.24 \\
\hline Mask application & 0.90 \\
\hline Total & $\mathbf{3 . 4 9}$ \\
\hline
\end{tabular}

$$
\begin{gathered}
\operatorname{Var}\left(\mathrm{R}_{\mathrm{i}}\right)=\sum_{\mathbf{v} \in \mathrm{R}_{\mathrm{i}}}\left\{\left[\left(\frac{\mathrm{v}_{\mathrm{x}}}{|\mathbf{v}|}-\frac{\mathrm{m}_{\mathrm{x}}}{|\mathbf{m}|}\right)^{2}+\left(\frac{\mathrm{v}_{\mathrm{y}}}{|\mathbf{v}|}-\frac{\mathrm{m}_{\mathrm{y}}}{|\mathbf{m}|}\right)^{2}\right] \cdot|\mathbf{v}|^{2}\right\} \\
\mathrm{m}_{\mathrm{x}}=\frac{1}{\mathrm{n}_{\mathrm{i}}} \sum_{\mathbf{v} \in \mathrm{R}_{\mathrm{i}}} \mathrm{v}_{\mathrm{x}}, \mathrm{m}_{\mathrm{y}}=\frac{1}{\mathrm{n}_{\mathrm{i}}} \sum_{\mathbf{v} \in \mathrm{R}_{\mathrm{i}}} \mathrm{v}_{\mathrm{y}}, \mathrm{m}=\left[\mathrm{m}_{\mathrm{x}}, \mathrm{m}_{\mathrm{y}}\right],
\end{gathered}
$$

It should be noted that the region variance has not been normalized with respect to the number of elements, so that large regions are implicitly penalized.

The above formula can be computationally simplified by means of some simple mathematical transformations:

$$
\operatorname{Var}\left(\mathrm{R}_{\mathrm{i}}\right)=2\left(\sum_{\mathbf{v} \in \mathrm{R}_{\mathrm{i}}}|\mathbf{v}|^{2}-\frac{\mathrm{s}_{\mathrm{x}} \sum_{\mathbf{v} \in \mathrm{R}_{\mathrm{i}}}|\mathbf{v}| \mathrm{v}_{\mathrm{x}}+\mathrm{s}_{\mathrm{y}} \sum_{\mathbf{v} \in \mathrm{R}_{\mathrm{i}}}|\mathbf{v}| \mathrm{v}_{\mathrm{y}}}{\sqrt{\mathrm{s}_{\mathrm{x}}^{2}+\mathrm{s}_{\mathrm{y}}^{2}}}\right),
$$

where $s_{x}=\sum_{\mathbf{v} \in R_{i}} v_{x}$ and $s_{y}=\sum_{\mathbf{v} \in R_{i}} v_{y}$.

\section{REFERENCES}

[1] J.D. Bowen, "The Home Office Automatic Fingerprint Pattern Classification Project," Proc. IEE Colloquiym Neural Network for Image Processing Applications, 1992.

[2] H. Bunke and G. Allermann, "Inexact Graph Matching for Structural Pattern Recognition," Pattern Recognition Letters, vol. 1, no. 5, pp. 245-253, 1983.

[3] G.T. Candela and R. Chellappa, "Comparative Performance of Classification Methods for Fingerprints," NIST Technical Report NISTIR 5163, Apr. 1993.

[4] G.T. Candela et al., "PCASYS-A Pattern-Level Classification Automation System for Fingerprints," NIST Technical Report NISTIR 5647, Aug. 1995.

[5] M.M.S. Chong et al., "Geometric Framework for Fingerprint Image Classification," Pattern Recognition, vol. 30, no. 9, pp. 1,475$1,488,1997$.
[6] M.J. Donahue and S.I. Rokhlin, "On the Use of Level Curves in Image Analysis," Image Understanding, vol. 57, no. 3, pp. 185-203, 1993.

[7] F. Galton, Finger Prints. London: McMillan, 1892.

[8] E.R. Henry, Classification and Uses of Finger Prints. London: Routledge, 1900

[9] P.A. Hughes and A.D.P. Green, "The Use of Neural Network for Fingerprint Classification," Proc. Second Int'l Conf. Neural Network, pp. 79-81, 1991.

[10] A.K. Jain, Fundamentals of Digital Image Processing, pp. 163-174. Prentice Hall, 1989.

[11] I.T. Jolliffe, Principle Component Analysis. New York: Springer-Verlag, 1986.

[12] M. Kamijo, "Classifying Fingerprint Images Using Neural Network: Deriving the Classification State," Proc. Third Int'l Conf. Neural Network, pp. 1,932-1,937, 1993.

[13] K. Karu and A.K. Jain, "Fingerprint Classification," Pattern Recognition, vol. 29, no. 3, pp. 389-404, 1996.

[14] M. Kawagoe and A. Tojo "Fingerprint Pattern Classification," Pattern Recognition, vol 17, no. 3, pp. 295-303, 1984.

[15] H.C. Lee and R.E. Gaensslen, Advances in Fingerprint Technology. Elsevier, 1991.

[16] A. Lumini, D. Maio, and D. Maltoni, "Continuous vs Exclusive Classification for Fingerprint Retrieval," Pattern Recognition Letters, vol. 18, no. 10, pp. 1,027-1,034, 1997.

[17] D. Maio, D. Maltoni, and S. Rizzi, "Topological Clustering of Maps Using a Genetic Algorithm," Pattern Recognition Letters, vol. 16, no. 1, pp. 89-96, 1995.

[18] D. Maio and D. Maltoni, "A Structural Approach to Fingerprint Classification," Proc. 13th ICPR, Vienna, Aug. 1996.

[19] D. Maio, D. Maltoni, and S. Rizzi, "Dynamic Clustering Of Maps In Autonomous Agents," IEEE Trans. Pattern Analysis and Machine Intelligence, vol. 18, no. 11, pp. 1,080-1,091, Nov. 1996.

[20] B. Moayer and K.S. Fu, "A Syntactic Approach to Fingerprint Pattern Recognition," Pattern Recognition, vol. 7, pp. 1-23, 1975.

[21] K. Moscinska and G. Tyma, "Neural Network Based Fingerprint Classification," Proc. Third Int'l Conf. Neural Network, pp. 229-232, 1993.

[22] K. Rao and K. Balck, "Type Classification of Fingerprints: A Syntactic Approach," IEEE Trans. Pattern Analysis and Machine Intelligence, vol. 2, no. 3, pp. 223-231, 1980.

[23] A. Senior, "A Hidden Markov Model Fingerprint Classifier," Proc. 31st Asilomar Conf. Signals, Systems, and Computers, pp. 306-310, 1997. 
[24] D.F. Specht, "Probabilistic Neural Network," Neural Networks, vol. 3, no. 1, pp. 109-118, 1990.

[25] V.S. Srinivasan and N.N. Murthy, "Detection of Singular Points in Fingerprint Images," Pattern Recognition, vol. 25, no. 2, pp. 139153, 1992.

[26] R.M. Stock and C.W. Swonger, "Development and Evaluation of a reader of Fingerprint Minutiae," Cornell Aeronautical Laboratory, Technical Report CAL no. XM-2478-X-1:13-17, 1969.

[27] C.I. Watson and C.L. Wilson, Nist Special Database 4, Fingerprint Database. U.S. Nat'1 Inst. of Standards and Technology, 1992.

[28] C.I. Watson, Nist Special Database 14, Fingerprint Database. U.S. Nat'l Inst. of Standards and Technology, 1993.

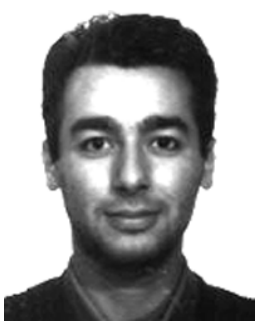

Raffaele Cappelli received the degree in computer science from the University of Bologna, Italy, in 1998. Since November 1998, he has been a PhD student at DEIS of the University of Bologna, with the research theme "Image Indexing and Retrieval." His research interests include also biometric systems and pattern recognition.

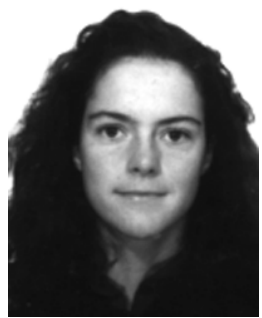

Alessandra Lumini received the degree in computer science from the University of Bologna, Italy, in 1996. Since November 1997, she has been a PhD student at DEIS of the University of Bologna, with the research theme "Image Databases." Her research interests include biometric systems, digital image watermarking, and spatial data structures. She is an IAPR member.

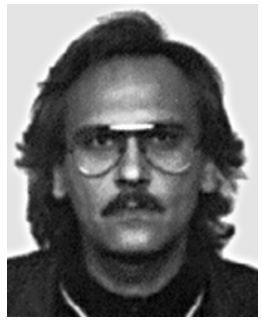

Dario Maio received the degree in electronic engineering from the University of Bologna in 1975. He is a full professor in the Computer Science Department, University of Bologna, Italy. $\mathrm{He}$ has published in the fields of distributed computer systems, computer performance evaluation, database design, information systems, neural networks, biometric systems, autonomous agents. Before joining the Computer Science Department, he received a fellowship from the C.N.R. (Italian National Research Council) for participation in the Air Traffic Control Project. He is an IEEE member. $\mathrm{He}$ is with CSITE-C.N.R. and with DEIS; he also teaches database and information systems in the Computer Science Dept., Cesena.

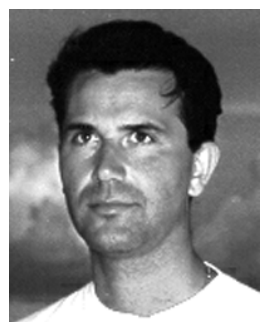

Davide Maltoni received the degree in computer science from the University of Bologna, Italy, in 1993. In 1998, he received his $\mathrm{PhD}$ in computer science and electronic engineering from DEIS, University of Bologna, with the research theme "Biometric Systems." $\mathrm{He}$ is an associate researcher in the Computer Science Department, University of Bologna, Italy. His research interests include also autonomous agents, pattern recognition, and neural nets. He is an IAPR member. 